\title{
Coupling Superposed 1D and 2D Shallow-Water Models: Source Terms in Finite Volume Schemes
}

\author{
E.D. Fernández-Nieto ${ }^{\text {a }}$ J. Marin $^{\mathrm{b}}$ J. Monnier ${ }^{\mathrm{c}, *}$ \\ ${ }^{a}$ Departamento de Matemática Aplicada I, Universidad de Sevilla. E.T.S. \\ Arquitectura. Avda, Reina Mercedes, s/n. 41012 Sevilla, Spain \\ ${ }^{\mathrm{b}}$ Univ. Grenoble 83 INRIA, Lab. LJK, B.P. 53, F-38041 Grenoble Cedex 9, France \\ ${ }^{\mathrm{c}}$ Univ. Toulouse - INSA, Institut de Mathématiques, F-31077 Toulouse cedex 4, \\ France
}

\begin{abstract}
We study the superposition of $1 \mathrm{D}$ and $2 \mathrm{D}$ shallow-water equations with non-flat topographies, in the context of river - flood modeling. Since we superpose both models in the bi-dimensional areas, we focus on the definition of the coupling term required in the $1 \mathrm{D}$ equations. Using explicit finite volume schemes, we propose a definition of the discrete coupling term leading to schemes globally well-balanced (the global scheme preserves water at rest whatever if overflowing or not). For both equations (1D and 2D), we can consider independent finite volume schemes based on well-balanced Roe, HLL, Rusanov or other scheme, then the resulting global scheme remains well-balanced. We perform a few numerical tests showing on the one hand the well-balanced property of the resulting global numerical model, on the other hand the accuracy and robutness of our superposition approach. Therefore, the definition of the coupling term we present allows to superpose a local 2D model over a 1D main channel model, with non-flat topographies and mix incoming-outgoing lateral fluxes, using independent grids and finite volume solvers.
\end{abstract}

Key words: shallow-water equations, superposition, coupling, finite volume, well-balanced, river, flood plain.

* Corresponding author: Jerome.Monnier@insa-toulouse.fr 


\section{Introduction}

In river hydraulics, operational models are generally based on the Saint-Venant equations (1D shallow-water). If overflowing, flood plain are represented in the 1D model by storage areas, that are defined by using empirical laws and/or terms to be calibrated, see [6] or e.g. [14]. Obviously, flow dynamic inside the storage areas is not computed; also the empirical laws can be difficult to calibrate. If for any reason, the end-user has to model the flow in the flood plain, a 2D model must be used. Then, the classical approach is to decompose the domain, re-define the mesh, then couple the 1D model (in the non-flooded areas) with 2D models (in flooded areas) at interfaces, see e.g. [15,13]. Coupling conditions have to be imposed at interfaces only. An efficient coupling procedure may be a Schwarz-like algorithm. Nevertheless, this approach presents some drawbacks. It requires to re-define the 1D hydraulic model (mesh, boundaries etc) and very probably the related topography data. The 1D model (which is potentially a complex network) must be segmented (decomposed) in order to combine it with the $2 \mathrm{D}$ models. It can be a heavy task.

A superposition approach is proposed in [8] and [12]. In such an approach, instead of decomposing the original 1D (network) model, one superposes the 2D model (socalled "local zoom model"). The superposition approach presents some advantages. The original 1D model remains intact and the 2D local models can be performed with their own dynamics (typically, time steps and mesh grids are much smaller for 2D solvers than for 1D solvers). Nevertheless, an accurate definition of the coupling terms between both models is required. At interfaces, incoming characteristics are still good conditions, but along the 1D main channel one must introduce a coupling term in the 1D equations (modelling the loss or gain of mass and momentum). This coupling term has to take into account the outgoing and incoming fluxes if overflowing. From a continuous point of view, the coupling source term can be derived formally from the 3D Navier-Stokes equations, see [12].

Then, next step is to define a stable and well-balanced global scheme. An important difficulty is to discriminate between the 1D-topography graph $Z_{b}(\tilde{x})$ and the 2D-topography graph $z_{b}(x, y)$, since $Z_{b}$ depends on the curvilinear coordinate $\tilde{x}$ while $z_{b}$ depends on the cartesian coordinates $(x, y)$. In addition for real cases, data are sparse, uncertain, and the 1D topography and the 2D topography do not have to respect the same hydrological constraints. If in addition, one wants to consider different meshes and schemes for the 1D model and the 2D model, the discretization of the coupling source term must be such that it leads to a consistent, stable and well-balanced scheme. This is the problem we address in the present study, while focusing on explicit finite volume schemes.

Let us point out that we present the $1 \mathrm{D}-2 \mathrm{D}$ coupling in the context of river hydraulics, but this could also be apply to any other flows involving $1 \mathrm{D}$ and 2D shallow-water equations with non-flat topographies.

We consider the possibility of using different finite volume schemes for the 1D model 
and the 2D model. In the numerical analysis presented in next sections, they can be based on different time-space grids but they must be explicit in time. More precisely, we consider finite volume methods in conservative form with source terms (the topography terms and the coupling term). Then, for both models, we can consider any solver belonging to a whole familly of approximate Riemann solvers. We prove that the resulting global scheme is well-balanced in the sense that it preserves water at rest, with and without overflowing.

We present some numerical results for an academic test case with a non-constant topography in which there are outgoing and incoming lateral fluxes. In order to couple the models, we use a Schwarz coupling algorithm (global in time). This could be done also by using an optimal control approach as in [8], [12]. The numerical results show that after convergence, the coupling source term $\Psi$ defined in the present study leads to a global solution as accurate as a full 2D solution in case of matching grids, and leads to a robust and accurate solution if grids are mismatching.

The paper is organized as follows. In Section 2, we present the two mathematical models. Their discretization using well-balanced finite volume scheme is presented in Section 3. The discretization of the coupling source term in 1D equations is described in Section 4. We begin with the simplest case (matching grids and 1D linear axis). Then, we consider the case of 1D curvilinear geometry with matching grids. Finally, the most general case (curvilinear and mismatching grids) is considered. We prove in Theorem 1 that the here introduced discrete source term leads to a global wellbalanced scheme, whatever the choice of the well-balanced finite volume method used for the $1 \mathrm{D}$ and 2D models. In Section 5 we present some numerical experiments to validate the definition of the discrete source term, and to show the efficiency of the present superposition approach. We recall briefly in Appendix A the derivation of the coupling source term in the 1D equations from the 3D Navier-Stokes equations (we refer to [12] for more details).

\section{Mathematical models}

\subsection{The 1D model with source term}

The 1D model is based on Saint-Venant equations (1D shallow-water equations). Nevertheless, since our goal is to couple this 1D model to a 2D shallow-water model, we must take into account transfers through the two lateral boundaries of the main channel. If we integrate the 3D Navier-Stokes equations over the vertical wetted area, in the presence of lateral transfer terms, we obtain some source terms in the 1D equations. The derivation of these source terms is presented in Appendix A. 
The result is the following. Let us denote the channel-following coordinates by: $\tilde{x}$. We denote the unidimensional variables (i.e. depending on $(\tilde{x}, t)$ only) as follows: $S$ the wet cross section, $Q$ the discharge, $H$ the water depth. And $\mathcal{Z}_{b}$ denotes the unidimensional topography (depending on $\tilde{x}$ only). We assume that: the channel width variations are small, $u$ is nearly constant over the cross section, and $(u, v)$ does not depend on $z$ on boundaries $b_{1}$ and $b_{2}$. Furthermore, for the sake of simplicity, we consider rectangular cross sections only. Hence $S=b . H$, where $b$ is the channel width, see Fig. A.3.

The derivation of the 1D shallow-water equations with source term is presented in Appendix A (replace $x$ by $\tilde{x}$ ). Under the assumptions above, the equations are the following:

$$
\left\{\begin{array}{l}
\frac{\partial S}{\partial t}+\frac{\partial Q}{\partial \tilde{x}}=-\left(q_{\eta_{1}}+q_{\eta_{2}}\right) \\
\frac{\partial Q}{\partial t}+\frac{\partial}{\partial \tilde{x}}\left(\frac{Q^{2}}{S}+P\right)-g \frac{\partial b}{\partial \tilde{x}} \frac{H^{2}}{2}+g S \frac{\partial \mathcal{Z}_{b}}{\partial \tilde{x}}=-\left(q_{\eta_{1}} u_{t_{1}}+q_{\eta_{2}} u_{t_{2}}\right)
\end{array}\right.
$$

where $P=g S \frac{H}{2}$ is a pressure term. In the right hand side, $q_{\eta_{i}}$ represents the discharge normal to the lateral boundary $i$ of the main channel, $i=1,2 ; u_{t_{i}}$ represents the tangential velocity at lateral boundary $i$, see Fig. A.4. (It is the projection of the horizontal velocity vector $(u, v)$ on the lateral boundary direction).

System (1) is closed with appropriate boundary conditions and initial conditions.

If we set $w=[S, Q]^{T}$ the unknown vector, then the $1 \mathrm{D}$ model writes as an hyperbolic system with source term:

$$
\partial_{t} w+\partial_{\tilde{x}} f_{1}(b(\tilde{x}), w)=g_{1}(\tilde{x}, w) \partial_{\tilde{x}} \mathcal{Z}_{b}+g_{2}(\tilde{x}, w) \partial_{\tilde{x}} b+\Psi
$$

where

$$
f_{1}(b(\tilde{x}), w)=\left(\begin{array}{c}
Q \\
\frac{Q^{2}}{S}+g \frac{S^{2}}{2 b}
\end{array}\right), \quad g_{1}(\tilde{x}, w)=\left(\begin{array}{c}
0 \\
-g S
\end{array}\right), \quad g_{2}(\tilde{x}, w)=\left(\begin{array}{c}
0 \\
\frac{g S^{2}}{2 b^{2}}
\end{array}\right)
$$

and

$$
\Psi=\left(\begin{array}{c}
-\left(q_{\eta_{1}}+q_{\eta_{2}}\right) \\
-\left(q_{\eta_{1}} u_{t_{1}}+q_{\eta_{2}} u_{t_{2}}\right)
\end{array}\right)
$$

is the coupling term in the $1 \mathrm{D}$ model i.e. normal discharges and tangential velocities normal to the (two) lateral boundaries of the main channel are provided by the $2 \mathrm{D}$ model. 


\subsection{The $2 D$ model}

The 2D hydraulics model is based on the bidimensional shallow-water equations in their conservative formulation. The unknowns are the water depth $h$ and the local discharge $\mathbf{q}=h \mathbf{u}$, where $\mathbf{u}=(u, v)^{T}$ is the depth-averaged velocity vector. In a bi-dimensional domain $\Omega$ and for a computational time interval $[0, T]$, equations are:

$$
\left\{\begin{array}{lr}
\partial_{t} h+\operatorname{div}(\mathbf{q})=0 & \text { in } \Omega \times] 0, T] \\
\partial_{t} \mathbf{q}+\operatorname{div}\left(\frac{1}{h} \mathbf{q} \otimes \mathbf{q}\right)+\frac{1}{2} g \nabla h^{2}+g h \nabla z_{b}+g \frac{n^{2}\|\mathbf{q}\|_{2}}{h^{7 / 3} \mathbf{q}=} & 0 \text { in } \Omega \times] 0, T]
\end{array}\right.
$$

where $g$ is the magnitude of the gravity, $z_{b}$ the (bi-dimensional) topography and $n$ the Manning roughness coefficient. Initial conditions $h(0)=h_{0}, \mathbf{q}(0)=\mathbf{q}_{0}$, and boundary conditions are given.

In all the sequel, $c=\sqrt{g h}$, denotes the local wave celerity.

Remark 2.1 a) Let us point out that we have to discriminate between the $1 D$ topography graph $Z_{b}(\tilde{x})$ and the 2D-topography graph $z_{b}(x, y)$. As a matter of fact, $Z_{b}$ depend on the curvilinear coordinate while $z_{b}$ depends on the cartesian coordinates. Furthermore, for actual data, the $1 D$ topography and the $2 D$ topography do not have to respect the same hydraulic constraints.

b) If the $2 D$ equations are locally coupled to $1 D$ equations (the $2 D$ model is playing the role of a zoom) then appropriate conditions at interfaces $1 D$-2D can be incoming characteristics, see e.g. [8,12].

If we define the unknown vector $W=W(t, \vec{x})$ with $\vec{x}=(x, y)$ by $W=\left(h, q_{x}, q_{y}\right)^{T}$, $\mathbf{q}=\left(q_{x}, q_{y}\right)$, then the system can be written as a $2 \mathrm{D}$ hyperbolic system with source terms. By simplicity we will not consider the friction term $g \frac{n^{2}\|\mathbf{q}\|_{2}}{h^{7 / 3}} \mathbf{q}$ in this study so that (4) can be rewritten as follows :

$$
\partial_{t} W+\partial_{x} F_{1}(W)+\partial_{y} F_{2}(W)=\kappa_{1}(\vec{x}, W) \partial_{x} z_{b}+\kappa_{2}(\vec{x}, W) \partial_{y} z_{b}
$$

where

$$
F_{1}(W)=\left(\begin{array}{c}
q_{x} \\
\frac{q_{x}^{2}}{h}+\frac{1}{2} g h^{2} \\
\frac{q_{x} q_{y}}{h}
\end{array}\right), \quad F_{2}(W)=\left(\begin{array}{c}
q_{y} \\
\frac{q_{x} q_{y}}{h} \\
\frac{q_{y}^{2}}{h}+\frac{1}{2} g h^{2}
\end{array}\right)
$$


and

$$
\kappa_{1}(W)=\left(\begin{array}{c}
0 \\
-g h \\
0
\end{array}\right), \quad \kappa_{2}(W)=\left(\begin{array}{c}
0 \\
0 \\
-g h
\end{array}\right)
$$

\section{Well-balanced finite volume methods}

We present the finite volume schemes considered to discretize the $1 \mathrm{D}$ and $2 \mathrm{D}$ models. First the numerical schemes for the $2 \mathrm{D}$ case are presented, in terms of some 1D numerical flux functions. Then, we present the numerical schemes for the one dimensional system. All the schemes considered here are based on well-balanced finite volume methods.

\subsection{Finite volume methods for the 2D system}

For the two-dimensional system, we consider a partition of the $2 \mathrm{D}$ domain $\Omega$ in control volumes denoted by $K_{i}$. By $E_{i j}$ we denote the common edges between the control volumes $K_{i}$ and its neighbour number $j ; \eta_{i j}$ is the unit normal vector to $E_{i j}$ outward to the control volume $K_{i}$, see Figure A.5. We also denote by $\left|K_{i}\right|$ the area of $K_{i},\left|E_{i j}\right|$ the length of $E_{i j}$ and

$$
W_{i}^{n} \approx \frac{1}{\left|K_{i}\right|} \int_{K_{i}} W\left(t^{n}, x, y\right) d x d y
$$

with $t^{n}=t^{n-1}+\Delta t$, being $\Delta t$ the time step considered for the 2D finite volume method.

The structure of 2D finite volume schemes is as follows:

$$
W_{i}^{n+1}=W_{i}^{n}-\frac{\Delta t}{\left|K_{i}\right|} \sum_{j \in K_{i}}\left|E_{i j}\right| \Phi^{2 D}\left(W_{i}^{n}, W_{j}^{n}, \eta_{i j}\right)+\Delta t G_{i}^{n}
$$

where

$$
G_{i} \approx \frac{1}{\left|K_{i}\right|} \int_{K_{i}}\left(\kappa_{1} \partial_{x} z_{b}+\kappa_{2} \partial_{y} z_{b}\right) d x d y .
$$

and $\Phi_{i j}^{2 D}=\Phi^{2 D}\left(W_{i}^{n}, W_{j}^{n}, \eta_{i j}\right)$ is an approximation of the normal flux related to the edge $E_{i j}$. 
The basic principle of $2 \mathrm{D}$ finite volume methods is to neglect tangential variations and to consider a projected 1D Riemann problem. Concretely, one starts from the $2 \mathrm{D}$ equations in the form $(5)$.

If we denote: $\mathcal{F}=\left(F_{1}, F_{2}\right), \kappa=\left(\kappa_{1}, \kappa_{2}\right)$, if $\eta=\left(\eta_{1}, \eta_{2}\right)$ is an unit vector, and $\eta^{\perp}=\left(-\eta_{2}, \eta_{1}\right)$, then we can rewrite the previous system as follows:

$$
\partial_{t} W+\partial_{\eta}(\mathcal{F} \cdot \eta)+\partial_{\eta^{\perp}}\left(\mathcal{F} \cdot \eta^{\perp}\right)=(\kappa \cdot \eta) \partial_{\eta} z_{b}+\left(\kappa \cdot \eta^{\perp}\right) \partial_{\eta^{\perp}} z_{b}
$$

Moreover, we can use the invariance rotation property of shallow-water equations (see [16]):

$$
(\mathcal{F} \cdot \eta)(W)=T_{\eta}^{-1} F_{1}\left(T_{\eta} W\right)
$$

where $T_{\eta}$ is the rotation matrix:

$$
T_{\eta}=\left(\begin{array}{ccc}
1 & 0 & 0 \\
0 & \eta_{1} & \eta_{2} \\
0 & -\eta_{2} & \eta_{1}
\end{array}\right)
$$

Using this invariance rotation property and multiplying the previous system by $T_{\eta}$ we obtain:

$$
\partial_{t}\left(T_{\eta} W\right)+\partial_{\eta} F_{1}\left(T_{\eta} W\right)+T_{\eta} \partial_{\eta^{\perp}}\left(\mathcal{F} \cdot \eta^{\perp}\right)=T_{\eta}(\kappa \cdot \eta) \partial_{\eta} z_{b}+T_{\eta}\left(\kappa \cdot \eta^{\perp}\right) \partial_{\eta^{\perp}} z_{b}
$$

The simplification of 2D finite volume method consists in neglecting the tangential variations. With this simplification, and by noticing that:

$$
T_{\eta}(\kappa \cdot \eta)=\kappa_{1}
$$

we obtain the 1D shallow-water equations plus a passive linear transport equation over the direction $\eta$ :

$$
\partial_{t}\left(T_{\eta} W\right)+\partial_{\eta} F_{1}\left(T_{\eta} W\right)=\kappa_{1}\left(T_{\eta} W\right) \partial_{\eta} z_{b}
$$

where: $T_{\eta} W=\left(h, q_{\eta}, q_{\eta^{\perp}}\right)^{T}$. The third equation of this system writes as follows:

$$
\partial_{t} q_{\eta^{\perp}}+\partial_{\eta}\left(\frac{q_{\eta} q_{\eta^{\perp}}}{h}\right)=0
$$

Then, in order to approximate the normal flux of the 2D shallow-water equations, $(F \cdot \eta)$, we consider numerical fluxes of the form:

$$
\Phi^{2 D}\left(W_{i}, W_{j}, \eta_{i j}\right)=T_{\eta_{i j}}^{-1} \Phi\left(T_{\eta_{i j}} W_{i}, T_{\eta_{i j}} W_{j}\right) .
$$


where $\Phi\left(V_{i}, V_{j}\right)$ is a numerical flux associated to the following 1D Riemann problem:

$$
\left\{\begin{array}{c}
\partial_{t} V+\partial_{\xi} F_{1}(V)=\kappa_{1}(V) \partial_{\xi} z_{b} \\
V(\xi, 0)=\left\{\begin{array}{l}
V_{1} \text { if } \xi<0 \\
V_{2} \text { if } \xi>0
\end{array}\right.
\end{array}\right.
$$

Let us point out that the 1D numerical flux $\Phi$ depends on the source term $\kappa_{1}(W) \partial_{\xi} z_{b}$ (i.e. $\Phi$ is not a numerical flux function corresponding to the homogeneous problem).

In the numerical schemes considered here the expression of $G_{i}$ is the following:

$$
G_{i}=\frac{1}{\left|K_{i}\right|} \sum_{j \in K_{i}} \frac{\left|E_{i j}\right|}{2} T_{\eta_{i j}}^{-1} \kappa_{1, i j}\left(\bar{z}_{b, j}-z_{b, i}\right)
$$

where $\kappa_{1, i j}=\kappa_{1}\left(\frac{V_{i}+V_{j}}{2}\right)$. The definition of $\bar{z}_{b, j}$ takes into account if flood is produced or not, since its definition includes a treatment of wet/dry fronts. Following [3], we propose to define it as follows:

$$
\bar{z}_{b, j}= \begin{cases}z_{b, j} & \text { if } h_{j} \neq 0 \\ z_{b, i}+h_{i} & \text { if } h_{j}=0\end{cases}
$$

Different approximate Riemann solvers. We denote by $\Phi$ the numerical flux function, corresponding to the $1 \mathrm{D}$ system (11).

We consider the following family of numerical solvers (see e.g. [5]):

$$
\Phi_{i j}=\Phi\left(V_{i}, V_{j}\right)=\frac{F_{1}\left(V_{j}\right)+F_{1}\left(V_{i}\right)}{2}-\frac{1}{2} P_{i j}\left\{\mathcal{A}_{i, j}\left(V_{j}-V_{i}\right)-\kappa_{1, i j}\left(\bar{z}_{b, j}-z_{b, i}\right)\right\}
$$

By $\mathcal{A}_{i, j}$ we denote the Roe matrix associated to $F_{1}$, such that:

$$
F_{1}\left(V_{j}\right)-F_{1}\left(V_{i}\right)=\mathcal{A}_{i j}\left(V_{j}-V_{i}\right)
$$

We suppose that matrix $\mathcal{A}_{i j}$ can be diagonalized, that is $\mathcal{A}_{i j}=X_{i j} \Lambda_{i j} X_{i j}^{-1}$, where $X_{i j}$ is a matrix whose columns are a basis of eigenvectors and $\Lambda_{i j}$ is the diagonal matrix defined by the eigenvalues. We denote the eigenvalues of $\mathcal{A}_{i, j}$ by: $\lambda_{1, i j}<\lambda_{2, i j}<\lambda_{3, i j}$.

Depending on the definition of $P_{i, j}$, we obtain different methods : 
- Roe method corresponds to $P_{i j}=X_{i j} \operatorname{sgn}\left(\Lambda_{i j}\right) X_{i j}^{-1}$ where:

$$
\operatorname{sgn}\left(\Lambda_{i j}\right)=\operatorname{diag}\left(\operatorname{sgn}\left(\lambda_{1, i j}\right), \operatorname{sgn}\left(\lambda_{2, i j}\right), \operatorname{sgn}\left(\lambda_{3, i j}\right)\right) .
$$

- HLL method corresponds to $P_{i j}=S_{1} I+S_{2} \mathcal{A}_{i j}^{-1}$, where:

$$
S_{1}=\frac{S_{r}+S_{l}}{S_{r}-S_{l}}, \quad S_{2}=\frac{-2 S_{r} S_{l}}{S_{r}-S_{l}}, \quad S_{l}=\min \left\{\lambda_{1, i j}, 0\right\}, \quad S_{r}=\max \left\{\lambda_{3, i j}, 0\right\}
$$

- Rusanov method corresponds to: $P_{i j}=\max _{(l=1,2,3)}\left\{\left|\lambda_{l, i j}\right|\right\} \mathcal{A}_{i j}^{-1}$.

- HLLC scheme. System (11) has a linearly degenerated field associated to the third equation (see (8), (9)), which corresponds to a passive linear transport.

To reduce the numerical diffusion on the approximation of the contact discontinuites, related to the linearly degenerated field, we can consider the HLLC scheme, see [16]. It is based on the definition of the physical flux function that verifies:

$$
\left[F_{1}\right]_{3}=\left[F_{1}\right]_{1} q_{\eta^{\perp}} / h
$$

Then, the third component of the numerical flux function is not defined by (13), but as a function of the first component of $\Phi$ and an upwind approximation of the passive scalar $\varphi^{*}$. We denote the resulting numerical flux by $\Phi^{c}$.

We consider the following extension of the HLLC method:

$$
\Phi_{i j}^{c}=\left(\begin{array}{c}
{\left[\Phi_{i j}\right]_{1}} \\
{\left[\Phi_{i j}\right]_{2}} \\
\left(\left[\Phi_{i j}\right]_{1}\right) \varphi_{i j}^{*}
\end{array}\right)
$$

where

$$
\varphi_{i j}^{*}=\left\{\begin{array}{l}
q_{\eta^{\perp} i} / h_{i} \text { if } S^{*}<0 \\
q_{\eta^{\perp} j} / h_{j} \text { if } S^{*}>0
\end{array}\right.
$$

and $\left[\Phi_{i j}\right]_{1}$ and $\left[\Phi_{i j}\right]_{2}$ are the two first components of the 1D flux defined by (13).

Although in HLLC method these two first components are given by the HLL method, any other solver defined by (13), as Roe or Rusanov, can be also used.

A consistent definition of the intermediate wave speed $S^{*}$ for not flat topography has been introduced in [7].

- Some other choices are possible; for example, flux limiter functions or Lax-Wendroff scheme (see [5]). 


\subsection{Finite volume methods for the $1 D$ system}

We describe the finite volume method considered to discretize the $1 \mathrm{D}$ hyperbolic system with source terms (2).

Let us denote by $\left\{\tilde{x}_{i+1 / 2}\right\}_{i=0}^{M}$ a set of points of the domain $[0, L]$ and $\tilde{x}_{i}=\left(\tilde{x}_{i-1 / 2}+\right.$ $\left.\tilde{x}_{i+1 / 2}\right) / 2$. The partition of the domain is defined by the set of control volumes $I_{i}=\left(\tilde{x}_{i-1 / 2}, \tilde{x}_{i+1 / 2}\right)$. We also denote $\left|I_{i}\right|=\left(\tilde{x}_{i+1 / 2}-\tilde{x}_{i-1 / 2}\right), \alpha_{i+1 / 2}=\left(\tilde{x}_{i+1}-\tilde{x}_{i}\right)$, and

$$
w_{i}^{n} \approx \frac{1}{\left|I_{i}\right|} \int_{I_{i}} w\left(t^{n}, \tilde{x}\right) d \tilde{x}
$$

with $t^{n}=t^{n-1}+\delta t$ ( $\delta t$ is the time step).

We consider finite volume methods in conservative form (see [2]) defined by :

$$
w_{i}^{n+1}=w_{i}^{n}-\frac{\delta t}{\left|I_{i}\right|}\left(\left(\phi_{i+1 / 2}\right)^{n}-\left(\phi_{i-1 / 2}\right)^{n}\right)+\delta t\left(g_{1 i}+g_{2 i}+\Psi_{i}\right) .
$$

where $g_{1 i}, g_{2 i}$ and $\Psi_{i}$ are second order approximations of $g_{1}(w) \partial_{\tilde{x}} Z_{b}, g_{2}(w) \partial_{\tilde{x}} b$ and $\Psi$ respectively:

$$
g_{1 i} \approx \frac{1}{\left|I_{i}\right|} \int_{I_{i}} g_{1}(w) \partial_{\tilde{x}} \mathcal{Z}_{b} d \tilde{x}, \quad g_{2 i} \approx \frac{1}{\left|I_{i}\right|} \int_{I_{i}} g_{2}(w) \partial_{\tilde{x}} b d \tilde{x}, \quad \Psi_{i} \approx \frac{1}{\left|I_{i}\right|} \int_{I_{i}} \Psi d \tilde{x} .
$$

We recall that $\Psi$ represents the coupling term. In the numerical schemes considered here the expression of $g_{l i}, l=1,2$, is the following:

$$
g_{l i}=\frac{g_{l}\left(w_{i-1 / 2}\right)+g_{l}\left(w_{i+1 / 2}\right)}{2}, \quad l=1,2 .
$$

For the considered 1D system, the flux function $f_{1}$ does not depend only on the vector of unknowns, but also on $b(\tilde{x})$. Therefore, the definition of the numerical flux function must take into account the derivative of the flux with respect to $b$, in order to obtain a well-balanced numerical scheme. Following [2], we consider the numerical scheme defined by:

$$
\begin{aligned}
& \phi_{i+1 / 2}=\frac{f_{1}\left(b_{i}, w_{i}\right)+f_{1}\left(b_{i+1}, w_{i+1}\right)}{2} \\
& -\frac{1}{2} P_{i+1 / 2}\left\{\mathcal{A}_{i+1 / 2}\left(w_{i+1}-w_{i}\right)-g_{1}\left(w_{i+1 / 2}\right)\left(\mathcal{Z}_{b, i+1}-\mathcal{Z}_{b, i}\right)\right. \\
& \left.-\left(g_{2}-\partial_{b} f_{1}\right)\left(w_{i+1 / 2}\right)\left(b_{i+1}-b_{i}\right)\right\} .
\end{aligned}
$$

And where $\mathcal{A}_{i+1 / 2}$ is the Roe matrix, verifying:

$$
f_{1}\left(b_{i+1 / 2}, w_{i+1}\right)-f_{1}\left(b_{i+1 / 2}, w_{i}\right)=\mathcal{A}_{i+1 / 2}\left(w_{i+1}-w_{i}\right) .
$$


Matrix $P_{i+1 / 2}$ is defined in terms of $\mathcal{A}_{i+1 / 2}$. The definition of matrix $P_{i+1 / 2}$ is similar to the definition of matrix $P_{i j}$, see equations (14)-(16). We have for example Roe, HLL and Rusanov methods.

\section{Coupling conditions and globally well-balanced schemes}

\subsection{Continuous coupling conditions}

The interactions between the 1D model and the 2D model are bilateral. The information exchange from the $1 \mathrm{D}$ model to the $2 \mathrm{D}$ one is done through boundary conditions at interfaces, see Fig.A.8. In case of river - local flood modelling for example, it is an open boundary condition problem. Since the 2D model is based on hyperbolic equations, it is natural to consider at interfaces the continuity of incoming characteristics, see e.g. [1]. In short, we impose that incoming characteristics in the $2 \mathrm{D}$ model are the same that those computed by the $1 \mathrm{D}$ model. If we denote by $W_{i}^{\Gamma_{k}}$ the i-th $1 \mathrm{D}$ incoming characteristic at boundary $\Gamma_{k}$ (see Fig. A.8), and $w_{i}$ the i-th 2D one, we impose:

$$
\int_{\Gamma_{k}} w_{i} d s=W_{i}^{\Gamma_{k}} ; \quad k \in\{3,4\}, i \in\{1,2\}
$$

We refer to [12] for detailed expressions of the characteristics variables.

The information exchange from the 2D model to the $1 \mathrm{D}$ model is done via the source term $\Psi$ defined by (3).

\subsection{Discrete source term and globally well-balanced schemes}

We present the definition of the discrete coupling source term $\Psi$. For a sake of clarity, we distinguish three cases.

First, we consider the simplest case: a linear 1D channel over the $x$ axis where the $1 \mathrm{D}$ channel width is exactly defined by the boundaries of the $2 \mathrm{D}$ mesh; in addition 1D mesh and 2D mesh are matching grids.

Second, we consider a more general case: the 1D channel is curvilinear, and takes into account the difference between the 1D mean (or analytical) channel and the real channel. Furthermore, we consider that the 1D mesh and 2D mesh are matching grids, see Fig. A.7. We present Theorem 4.1 stating that the resulting global scheme (1D-2D) is well-balanced, in the sense that it exactly preserves water at rest.

Third, we extend the definition to the most general case where meshes are not 
related each other, and different times steps can be used for the $1 \mathrm{D}$ and the 2D solvers. In the latter case, extension of Theorem 4.1 is straightforward to prove; proof is sketched.

\subsubsection{Meshes properties}

First, let us precise what we call a "general domain". The width of the 1D channel is defined as a mean value of the real channel, see Fig. A.7. This defines the so-called analytical channel. Its boundaries are symmetrical with respect to its middle curve. The latter is defined by:

$$
\gamma(\widetilde{x})=(x(\widetilde{x}), y(\widetilde{x})), \quad \widetilde{x} \in\left[\widetilde{x}_{0}, \widetilde{x}_{f}\right]
$$

Let $b(\widetilde{x})$ be the width function. Hence, the analytical 1D domain immersed in the $2 \mathrm{D}$ domain is defined by:

$$
\gamma_{1}(\widetilde{x})=\gamma(\widetilde{x})-\frac{b(\widetilde{x})}{2}\left(-y^{\prime}(\widetilde{x}), x^{\prime}(\widetilde{x})\right), \quad \text { and } \quad \gamma_{2}(\widetilde{x})=\gamma(\widetilde{x})+\frac{b(\widetilde{x})}{2}\left(-y^{\prime}(\widetilde{x}), x^{\prime}(\widetilde{x})\right)
$$

The mesh points matching with the 2D mesh are $\left\{\gamma\left(\widetilde{x}_{i+1 / 2}\right)\right\}_{i=1}^{N}$. The center of the volume $K_{i}$ can be approximated by $\gamma\left(\widetilde{x}_{i}\right)$ with $\widetilde{x}_{i}=\left(\widetilde{x}_{i-1 / 2}+\widetilde{x}_{i+1 / 2}\right) / 2$.

In each case (the so-called simplest case, matching case, and mismatching case), the 2D mesh $\mathcal{T}$ always contain a submesh, say $\tau$, composed by quadrangular cells that constitutes an approximation of the 1D channel. The boundary of every cell $K \in \tau$ is supposed to intersect the axis of the channel in two points belonging to two opposite edges, the two remaining edges being an approximations of the boundaries of the 1D channel. Moreover, if the point of the channel of coordinate $\tilde{x}$ belongs to $K \in \tau$ then $b(\tilde{x})$ is equal to the width of $K$ following the normal direction to the axis in $\gamma(\tilde{x})=(x(\tilde{x}), y(\tilde{x}))$.

- In the simplest case, the axis of the 1D channel is supposed to be the $x$-axis (hence $\tilde{x}=x$ ) and, given a cell of the $1 \mathrm{~d}$ mesh $\left[x_{i-1 / 2}, x_{i+1 / 2}\right]$ there exists a cell $K \in \tau$ whose intersection with the axis is equal to $\left[x_{i-1 / 2}, x_{i+1 / 2}\right]$, see Fig. A.6.

- In the general domain case with matching grids (so-called matching case), we consider the following geometrical layout. Given a cell of the 1D mesh $\left[\tilde{x}_{i-1 / 2}, \tilde{x}_{i+1 / 2}\right]$ there exists a cell $K \in \tau$ that contains the arc of the axis linking $\gamma\left(\tilde{x}_{i-1 / 2}\right)$, $\gamma\left(\tilde{x}_{i+1 / 2}\right)$. Moreover, $\gamma\left(\tilde{x}_{i-1 / 2}\right)$ and $\gamma\left(\tilde{x}_{i+1 / 2}\right)$ belongs to the boundary of $K$, see Fig. A.7.

- In the mismatching case, given a cell of the $1 \mathrm{~d}$ mesh $\left[\tilde{x}_{i-1 / 2}, \tilde{x}_{i+1 / 2}\right]$ there exists an ordered family of neighbor cells of $\tau, K_{i_{1}}, \ldots, K_{i_{m}}$ whose union contains the arc of the axis linking $\gamma\left(\tilde{x}_{i-1 / 2}\right), \gamma\left(\tilde{x}_{i+1 / 2}\right)$. Moreover, in the subgrid case, $\gamma\left(\tilde{x}_{i-1 / 2}\right)$ and $\gamma\left(\tilde{x}_{i+1 / 2}\right)$ belongs respectively to the intersection of $K_{i_{1}}$ and $K_{i_{m}}$ with the axis. 


\subsubsection{The simplest case with matching grids}

We assume that time grids for 1D and 2D models are the same: $\delta t=\Delta t$ and $t_{\tilde{m}}=t_{m}$. As precised above, we assume that the 1D main channel is rectilinear and parallel to the $x$-axis, see Fig. A.6. Moreover, the $1 \mathrm{D}$ channel is defined between: $y=-b(x) / 2$ and $y=b(x) / 2$; which corresponds exactly to the $2 \mathrm{D}$ mesh.

The discrete source term $\Psi_{i}$ must be an approximation of the continuous source term $\Psi$ :

$$
\Psi_{i} \approx \Psi=\left(\begin{array}{c}
-\left(q_{\eta_{1}}+q_{\eta_{2}}\right) \\
-\left(q_{\eta_{1}} u_{t_{1}}+q_{\eta_{2}} u_{t_{2}}\right)
\end{array}\right)
$$

Moreover, we can approximate $u_{t_{1}}$ by $u_{\eta_{1}^{\perp}}$ and $u_{t_{2}}$ by $\left(-u_{\eta_{2}^{\perp}}\right)$. And,

$$
\left[F_{1}\left(T_{\eta} W\right)\right]_{1}=q_{\eta}, \quad\left[F_{1}\left(T_{\eta} W\right)\right]_{3}=q_{\eta} u_{\eta^{\perp}} .
$$

where $u_{\eta^{\perp}}=q_{\eta^{\perp}} / h$. Then, we can defined $\Psi_{i}$ as an approximation of

$$
\left(\begin{array}{c}
-\left[F_{1}\left(T_{\eta_{1}} W\right)\right]_{1}-\left[F_{1}\left(T_{\eta_{2}} W\right)\right]_{1} \\
-\left[F_{1}\left(T_{\eta_{1}} W\right)\right]_{3}+\left[F_{1}\left(T_{\eta_{2}} W\right)\right]_{3}
\end{array}\right)
$$

As $\left[F_{1}\left(T_{\eta} W\right)\right]_{1}=-\left[F_{1}\left(T_{(-\eta)} W\right)\right]_{1}$ and $\left[F_{1}\left(T_{\eta} W\right)\right]_{3}=\left[F_{1}\left(T_{(-\eta)} W\right)\right]_{3}$, we can rewrite previous expression as

$$
-\left(\begin{array}{c}
\left.-\left[F_{1}\left(T_{\left(-\eta_{1}\right)} W\right]\right)\right]_{1} \\
{\left[F_{1}\left(T_{\left(-\eta_{1}\right)} W\right)\right]_{3}}
\end{array}\right)+\left(\begin{array}{c}
-\left[F_{1}\left(T_{\eta_{2}} W\right)\right]_{1} \\
{\left[F_{1}\left(T_{\eta_{2}} W\right)\right]_{3}}
\end{array}\right)
$$

Let us use the following notation: $\Phi\left(\eta_{i j}\right)=\Phi\left(T_{\eta_{i j}} W_{i}, T_{\eta_{i j}} W_{j}\right)$. For a fixed volume $K_{i}$ of the submesh of the $1 \mathrm{D}$ channel, indices $j_{1}$ and $j_{2}$ correspond to the volumes $K_{j_{1}}$ and $K_{j_{2}}$. The intersection between $K_{j_{1}}$ and $K_{j_{2}}$ with $K_{i}$ are contained in the opposite edges of the 1D channels (see Fig. A.6). Then, by taking into account that $\Phi$ is an approximation of the flux function $F_{1}$ at the corresponding boundary, we can set the following definition.

$$
\Psi_{i}=-\underline{\Psi}\left(-\eta_{i j_{1}}\right)+\underline{\Psi}\left(\eta_{i j_{2}}\right)=\sum_{k=1}^{2} s_{i j_{k}} \underline{\Psi}\left(s_{i j_{k}} \eta_{i j_{k}}\right),
$$

where

$$
\underline{\Psi}\left(\eta_{i j}\right)=\left(\begin{array}{c}
-\left[\Phi\left(\eta_{i j}\right)\right]_{1} \\
{\left[\Phi\left(\eta_{i j}\right)\right]_{3}}
\end{array}\right)
$$

and

$$
s_{i j_{k}}=-\operatorname{sgn}\left(\eta_{i j_{k}}^{\perp} \cdot(1,0)\right), \quad k=1,2 .
$$


Observe that $(1,0)$ is the direction of the axis of the $1 \mathrm{D}$ channel, for this simple case.

Now, we rewrite this expresion of $\underline{\Psi}\left(\eta_{i j}\right)$. This new form to rewrite the coupling term help us to define $\Psi_{i}$ for more complex cases.

First, observe that:

$$
\left[\Phi\left(\eta_{i j}\right)\right]_{l}=\left[T_{\eta_{i j}}\left(T_{\eta_{i j}}^{-1} \Phi\left(\eta_{1, j}\right)-\frac{1}{2} T_{\eta_{i j}}^{-1} \kappa_{1, i j}\left(\bar{z}_{b, j}-z_{b, i}\right)\right)\right]_{l}, \quad l=1,3,
$$

where, $\kappa_{1, i j}=\kappa_{1}\left(\left(W_{i}+W_{j}\right) / 2\right)$. Secondly,

$$
\left[T_{\eta^{\perp}} r\right]_{1}=\left[T_{\eta} r\right]_{1} \quad \text { and } \quad\left[T_{\eta^{\perp}} r\right]_{2}=-\left[T_{\eta} r\right]_{3}, \quad \forall r \in \mathbb{R}^{3} .
$$

Then, using also that $\Phi^{2 D}$ is defined in terms of $\Phi$ (see equation (10)), we obtain,

$$
\left[\underline{\Psi}\left(\eta_{i j}\right)\right]_{l}=-\left[T_{\eta_{i, j}^{\perp}}\left(\Phi^{2 D}\left(W_{i}, W_{j}, \eta_{i j}\right)-\frac{1}{2} T_{\eta_{i j}}^{-1} \kappa_{1, i j}\left(\bar{z}_{b, j}-z_{b, i}\right)\right)\right]_{l} \quad l=1,2 .
$$

Finally, we define:

$$
\left[\bar{\Psi}\left(\beta, \eta_{i j}\right)\right]_{l}=-\left[T_{\beta}\left(\Phi^{2 D}\left(W_{i}, W_{j}, \eta_{i j}\right)-\frac{1}{2} T_{\eta_{i j}}^{-1} \kappa_{1, i j}\left(\bar{z}_{b, j}-z_{b, i}\right)\right)\right]_{l} \quad l=1,2 .
$$

It verifies:

$$
\bar{\Psi}(\beta,(-\eta))=-\bar{\Psi}(\beta, \eta)
$$

Then, we can rewrite the definition of the coupling term $\Psi_{i}$ as

$$
\Psi_{i}=\bar{\Psi}\left(-\eta_{i j_{1}}^{\perp}, \eta_{i j_{1}}\right)+\bar{\Psi}\left(\eta_{i j_{2}}^{\perp}, \eta_{i j_{2}}\right)=\sum_{k=1}^{2} \bar{\Psi}\left(s_{i j_{k}} \eta_{i j_{k}}^{\perp}, \eta_{i j_{k}}\right) .
$$

\section{Remark 4.1}

(1) For $\Phi_{i j}^{2 D}=T_{\eta_{i j}}^{-1} \Phi_{i j}^{c}$ with $\Phi^{c}$ defined by (16), we have

$$
\left[\underline{\Psi}\left(\eta_{i j}\right)\right]_{2}=\left[\Phi\left(\eta_{i j}\right)\right]_{1} \varphi_{i j}^{*}
$$

where $\Phi_{i j}=\Phi\left(T_{\eta_{i j}} W_{i}, T_{\eta_{i j}} W_{j}\right)$ and

$$
\varphi_{i, j}^{*}= \begin{cases}\left(-q_{1 i} \eta_{2}+q_{2 i} \eta_{1}\right) / h_{i} & \text { if } S^{*}<0 \\ \left(-q_{1 j} \eta_{2}+q_{2 j} \eta_{1}\right) / h_{j} & \text { if } S^{*}>0\end{cases}
$$

Then, we observe that the tangential velocity $u_{t}$ in the continuous coupling term $\underline{\Psi}$ is approximated by $\left( \pm \varphi_{i j}^{*}\right)$. And $\varphi_{i, j}^{*}$ is an approximation of $\left(q_{i} / h_{i}\right) \cdot \eta_{i j}^{\perp}$ or $\left(q_{j} / h_{j}\right) \cdot \eta_{i j}^{\perp}$, depending on the sign of $S^{*}$. 
So, $u_{t}$ is approximated in terms of the sign of $S^{*} . S^{*}$ gives an approximation of the normal velocity to the edge. In other words, the present definition of the discrete coupling term includes an upwind approximation of the tangential velocity depending on the sign of the normal velocity at lateral boundary.

Classically, in 1D St-Venant models with source term (modelling over-flowing), tangential velocities $u_{t}$ at lateral boundaries are approximated by the $1 D$ channel velocity. Therefore, in case of incoming normal velocity into the $1 D$ channel (lateral filling), such an approximation would give an inconsistent or unstable scheme. At the contrary, the present 1D-2D coupling and the resulting coupling source term handle correctly with the filling case (in addition of the emptying - overflowing case). This feature can be crucial when modelling complex flows involving a 1D channel combined with flooded (or filled) $2 D$ areas.

(2) By using in the definition of $\left[\underline{\Psi}\left(\eta_{i j}\right)\right]_{2}$ some other numerical scheme defined by $\Phi_{i j}^{2 D}=T_{\eta_{i j}}^{-1} \Phi_{i j}$, we obtain different upwind approximations of $q_{\eta_{i j}} u_{\eta_{i j}^{\perp}}$, depending on the definition of $P_{i j}$.

(3) We can prove that the resulting global scheme (1D-2D) is well-balanced. Since it is a particular case of the forthcoming general case, we refer to next subsection for the proof (see Theorem 4.1 below).

\subsubsection{General domain with matching grids}

In the definition of $\bar{\Psi}(\beta, \eta)$, see $(22), \eta$ is associated to the computation of $q_{\eta}$ and vector $\beta$ to $u_{\beta}$. This allows us to study more general cases. In the present case, we consider the geometrical layout presented in subsection 4.2.1. Since the analytical 1D channel does not coincide with the boundaries of the real channel meshed in the 2 Deometry, we need to correct the previous formula of $\Psi_{i}$. Thus, we present below the formula of $\Psi_{i}$ which takes into account the difference between the analytical $1 \mathrm{D}$ channel and the real channel boundaries which are meshed. To this end, in the definition of $\Psi(\beta, \eta)$ we introduce $\beta$ in order to adjust $u_{\beta}$ to the tangent direction of the analytical 1D channel. In general cases, we have: $\beta \neq \pm \eta^{\perp}$.

As we pointed out in Remark 4.1, classically in the 1D St-Venant model, tangential velocities $u_{t}$ at lateral boundaries are approximated by the $1 \mathrm{D}$ channel velocity. Here since we compute the $2 \mathrm{D}$ velocity too, $u_{t}$ at lateral boundaries are defined as an upwind approximation of the projection of the 2D velocity onto the $1 \mathrm{D}$ channel boundaries.

For a given control volume $K_{i}$ we set:

$$
\beta_{i j_{1}}=\frac{\gamma_{1}^{\prime}\left(\widetilde{x}_{i}\right)}{\left|\gamma_{1}^{\prime}\left(\widetilde{x}_{i}\right)\right|}, \quad \beta_{i j_{2}}=\frac{\gamma_{2}^{\prime}\left(\widetilde{x}_{i}\right)}{\left|\gamma_{2}^{\prime}\left(\widetilde{x}_{i}\right)\right|},
$$

the unitary tangent vectors to the boundaries defined by $\gamma_{1}$ and $\gamma_{2}$, respectively. 
On the other hand, we remember that the definition of the coupling term is an approximation of:

$$
\left(\begin{array}{c}
-\left[F_{1}\left(T_{\eta} W\right)\right]_{1} \\
{\left[F_{1}\left(T_{\eta} W\right)\right]_{3}}
\end{array}\right)=\left(\begin{array}{c}
-\left[T_{\eta^{\perp}} T_{\eta}^{-1} F_{1}\left(T_{\eta} W\right)\right]_{1} \\
-\left[T_{\eta^{\perp}} T_{\eta}^{-1} F_{1}\left(T_{\eta} W\right)\right]_{2}
\end{array}\right) .
$$

The idea is to change $\eta^{\perp}$ by another vector $\beta$. We have:

$$
\left[-T_{\beta} T_{\eta}^{-1} F_{1}\left(T_{\eta} W\right)\right]_{1}=-q_{\eta} \quad \text { and } \quad\left[-T_{\beta} T_{\eta}^{-1} F_{1}\left(T_{\eta} W\right)\right]_{2}=-\left(q_{\eta} u_{\beta}+\frac{g}{2} h^{2} \beta \cdot \eta\right) .
$$

The vector $\beta$ is introduced in order to approximate $u_{t}$ by $u_{\beta}$. If $\beta= \pm \eta^{\perp}$ then $\left[-T_{\beta} T_{\eta}^{-1} F_{1}\left(T_{\eta} W\right)\right]_{3}=q_{\eta} u_{\beta}$; otherwise, it remains the term $\left(\frac{g}{2} h^{2} \beta \cdot \eta\right)$.

Hence, a correction must be introduced into the definition of $\Psi_{i}$ in order to rid of the term $\left(\frac{g}{2} h^{2} \beta \cdot \eta\right)$.

We have:

$$
\frac{g}{2} h^{2} \beta \cdot \eta=-\frac{h}{2}\left[T_{\beta} T_{\eta}^{-1} \kappa_{1}(W)\right]_{2} \quad \text { and } \quad\left[T_{\beta} T_{\eta}^{-1} \kappa_{1}(W)\right]_{1}=0 .
$$

So, we propose the following definition of the coupling term:

$$
\Psi_{i}=\widehat{\Psi}\left(\beta_{i j_{1}}, \eta_{i j_{1}}\right)+\widehat{\Psi}\left(\beta_{i j_{2}}, \eta_{i j_{2}}\right),
$$

with

$$
\left[\widehat{\Psi}\left(\beta, \eta_{i j}\right)\right]_{l}=-\left[T_{\beta}\left(\Phi^{2 D}\left(W_{i}, W_{j}, \eta_{i j}\right)-\frac{1}{2} T_{\eta_{i j}}^{-1} \kappa_{1, i j}\left(\bar{z}_{b, j}-z_{b, i}\right)+\frac{h_{i}}{2} T_{\eta}^{-1} \kappa_{1, i}\right)\right]_{l},
$$

for $l=1,2$ and $\kappa_{1, i}=\kappa_{1}\left(W_{i}\right)$. Of course, if $\beta= \pm \eta^{\perp}$ then $\widehat{\Psi}$ equals to $\bar{\Psi}$.

Remark 4.2 Let us recall that $1 D$ numerical flux is denoted by $\phi$, $2 D$ numerical flux by $\Phi^{2 D}$, and $\Phi^{2 D}=T_{\eta}^{-1} \Phi$ or $\Phi^{2 D}=T_{\eta}^{-1} \Phi^{c}$. $\Phi$ and $\Phi^{c}$ are $1 D$ numerical fluxes defined by (13) and (16) respectively.

We point out that definition (24) of the discrete coupling term does not imply any particular relationship between the $1 D$ and $2 D$ solvers involved. Therefore, it is possible to use for example low computational cost scheme for $2 D$ model (e.g. Rusanov method) with a more accurate scheme for $1 D$ model (e.g. Roe solver).

Let us state the well-balanced properties of the resulting global scheme with the proposed discrete coupling term (24). We have

Theorem 4.1 The scheme defined by (17)-(18) and the coupling term (24) exactly preserves water at rest with or without overflowing. This is true for any choice of the upwinding matrix $P_{i+1 / 2}$ for the $1 D$ solver (see (18)) and for any choice of the $2 D$ numerical solver. 


\section{PROOF.-}

As the 1D method is well-balanced (see [2]), it is enough to prove that in case of water at rest the coupling term vanish, independently if overflowing or not.

Let us define:

$$
\mathcal{C}=\frac{F_{1}\left(W_{j}\right)-F_{1}\left(W_{i}\right)}{2}-\frac{1}{2} \kappa_{1, i j}\left(\bar{z}_{b j}-z_{b i}\right)
$$

For water at rest, we have: $\mathcal{C}=0$.

The second term in the expression of $\mathcal{C}$ writes:

$$
\frac{g}{2} \frac{h_{j}+h_{i}}{2}\left(h_{j}+\bar{z}_{b j}-h_{i}-z_{i}\right)
$$

If $h_{j}=0$, in vertu of the definition of $\bar{z}_{b j}$ to treat dry/wet areas, see (12), we have: $\bar{z}_{b j}=z_{i}+h_{i}$. Hence this term equals to zero.

Otherwise, the stationary solution of water at rest verifies: $h+z_{b}=$ constant. Hence, again, this equals to zero.

Using the property of Roe matrix, the term that multiplies $P_{i j}$ in the definition of $\Phi_{i j}$, is $\mathcal{A}_{i j}^{-1} \mathcal{C}$. Hence, it is equal to zero.

So,

$$
\Phi_{i+1 / 2}=\frac{F_{1}\left(W_{i}\right)+F_{1}\left(W_{j}\right)}{2}
$$

and

$$
\left.\left[\widehat{\Psi}\left(\beta, \eta_{i j}\right)\right)\right]_{l}=-\left[T_{\beta} T_{\eta_{i j}}^{-1}\left(\frac{F_{1}\left(W_{i}\right)+F_{1}\left(W_{j}\right)}{2}-\frac{1}{2} \kappa_{1, i j}\left(\bar{z}_{b j}-z_{b i}\right)+\frac{h_{i}}{2} \kappa_{1, i}\right)\right]_{l},
$$

for $l=1,2$.

Finally, the term that multiplies $T_{\beta} T_{\eta_{i j}}^{-1}$ in the previous expression, coincides with $\mathcal{C}$ (defined by $(25)$ ); therefore it is equal to zero. Then, $\widehat{\Psi}\left(\beta, \eta_{i j}\right)=0$ and $\Psi_{i}=0$.

Remark 4.3 The present $2 D$ finite volume methods do not preserve all stationary solutions of the system. As a matter of fact, the $2 D$ finite volume methods neglect the tangential variations of the unknown at interfaces. Nevertheless, for the family of methods presented in Section 3, it is possible to prove that they preserve all regular $1 D$ stationary solutions, up to second order in $\Delta x$ (see [4]), if the finite volume mesh is defined by rectangles oriented in the same sense than the solution variations. Furthermore, we can prove the same property for the present coupled system. It consists to prove that $\Psi_{i}=\mathcal{O}\left(\Delta x^{2}\right)$ for any stationary solution. 


\subsubsection{Subgrid and mismatching grids cases}

In this section we consider the case of subgrid meshes or mismatching $1 \mathrm{D} / 2 \mathrm{D}$ grids, in the sense described in subsection 4.2.1. In both cases, the generalization of the source term $\Psi$ is straightforward. Let us define the projection operator $R$ that computes average values over $\tilde{K}_{i}$ and $\delta t$ :

$$
R_{i}\left(t_{m}, v\right)=\frac{1}{\delta t\left\|\tilde{K}_{i}\right\|} \int_{t_{m}}^{t_{m}+\delta t} \int_{\tilde{K}_{i}} v d \Omega d t
$$

where $\tilde{K}_{i}$ is a $2 \mathrm{D}$ volume defined as a subset matching with the $1 \mathrm{D}$ mesh. Remember that $\delta t$ is the time step corresponding to the discretization of the $1 \mathrm{D}$ model.

We consider a piecewise constant function, $F_{\Psi_{i}}(x, t)$ defined by the value $\left\{\Psi_{i j}\right\}_{j}$ at the volumes $K_{j}$. Thus, we define:

$$
\Psi_{i}^{n}=R_{i}\left(t_{n}, F_{\Psi_{i}}\right)
$$

The main difference between the subgrid case and mismatching one, is the computation of the previous integral. For the subgrid case, $\tilde{K}_{i}$ is the union of several volumes of the $2 \mathrm{D}$ mesh, then the integral is easily computed. In the mismatching case, $\tilde{K}_{i}$ is not exactly defined by the union of several volumes $K_{j}$, then it is necessary to estimate the corresponding intersection areas.

The integration in time in the denition $R_{i}\left(t_{m}, v\right)$ is due to the fact that the time steps may be different for the $1 \mathrm{D}$ and the $2 \mathrm{D}$ solvers.

The resulting scheme verifies the same well-balanced properties than for the previous cases. The proof is straightforward since each integrand vanishes for water at rest (with or without overflowing), as it has been seen in proof of Theorem 4.1. Then $\Psi_{i}^{n}=0$, and the well-balanced property is obtained.

\section{$5 \quad$ Numerical results}

We perform two types of numerical tests. Test 1 is a numerical verification of the well-balanced property when water is at rest. Test 2 concerns a steady-state flow (in a non-constant topography) involving incoming and outgoing lateral fluxes. The 1D solver is the HLL scheme, see (15), while the 2D solver is the corresponding HLLC scheme, see (16). These solvers are implemented into our software DassFlow $[11,10]$. The coupling algorithm used is a global in time Schwarz-like algorithm, see Fig. A.9. Inputs of the coupling algorithm are: initial conditions for both 1D and 2D models, boundary conditions of the 1D model and a first guess of boundary conditions for the $2 \mathrm{D}$ model. 


\subsection{Test 1. Water at rest}

The first test case considered corresponds to a water at rest solution with and without overflowing. All tests done are successfull since water stays at rest (norm of velocities are about $10^{-8}$ ). The used mesh is presented in Fig. A.10 (2274 cells).

\subsection{Test 2. Steady state solution with incoming - outgoing flow}

\subsubsection{Validation procedure}

The definition of a non trivial steady state analytical solution for the coupled model is a difficult task. We decided to use the following approach to validate our coupling process:

- First, we compute a "reference" steady flow using the validated 2D HLLC solver for the entire computational domain on a fine grid.

- Then, we use our coupling algorithm and compare the solution with the previous generated "reference" solution. For the 1D model, we use either the same fine grid (matching grids case) or a coarse grid (subgrids case).

\subsubsection{Test case description}

This test is presented in Fig. A.10. The channel length is $200 \mathrm{~m}$, the main channel is $2 \mathrm{~m}$ wide and the circle diameter $100 \mathrm{~m}$. The $2 \mathrm{D}$ mesh is made of triangular cells, except in the main channel where cells are rectangular. The bathymetry is the following:

$$
z_{b}(x, y)=\left\{\begin{array}{cc}
0.5 & \text { if } 80 \leq x \leq 120 \\
0 & \text { otherwise }
\end{array}\right.
$$

Boundary conditions are the following:

- Inflow (West) : discharge imposed $q_{i n}=2 \mathrm{~m}^{3} / \mathrm{s}$,

- Outflow (East) : $\frac{\partial h}{\partial \mathbf{n}}=\frac{\partial \mathbf{q}}{\partial \mathbf{n}}=0$,

- Walls boundary conditions are imposed on the other boundaries.

This test case includes a non-constant bathymetry, incoming lateral fluxes and outgoing ones. Thus, it is suitable to show the robustness of our definition of the coupling term $\Psi$. 
Using a time step $\Delta t=0.1$ (CFL number is about 0.1 ), we obtain the steady state presented in Fig. A.10 b). For the coupling test, the simulation time is $T=50 \mathrm{~s}$

\subsubsection{Matching grids case}

The 2D "reference mesh" (see Fig. A.10) is made of 2274 cells. Both 1D and "2D zoom" meshes are restriction of the "reference mesh" on the corresponding area.

Validation of the source term.

We validate the expression of the coupling source term (in the $1 \mathrm{D}$ equations) and its implementation as follows.

- Initial conditions for both the $1 \mathrm{D}$ and $2 \mathrm{D}$ models are extracted from the $2 \mathrm{D}$ "reference" solution.

- Boundary conditions of the 2D model are extracted from the 2D reference solution.

Therefore, the 2D zoom model must reproduce exactly the solution of the $2 \mathrm{D}$ reference model (in one iteration of Schwarz algorithm). Furthermore if $\Psi$, the coupling source term in 1D equations, is accurate, then the 1D model solution should fit perfectly with the reference solution. The numerical results show that both the 1D solution and the 2D zoom solution match perfectly with the reference solution (differences in percent are about $10^{-6}$ ). Thus, this test case shows the accuracy of the coupling source term $\Psi$ and validates its implementation.

Coupling with Schwarz algorithm.

Now, we consider the coupling algorithm of Schwarz described in Fig. A.9. The convergence threshold used is $\epsilon_{\text {Schwarz }}=10^{-11}$.

We have a-priori no guarantee that the coupling algorithm will converge to the $2 \mathrm{D}$ reference solution. It depends partially on the first guess for the $2 \mathrm{D}$ incoming characteristics (thus on the initial condition). Also, in the present test case, the flow computed is steady-state and the iterates are unsteady. In our numerical tests, starting from a "reasonable initial condition", the algorithm converged with 4 iterates at maximum.

We present in Fig. A.11 the three solutions: the reference one, the 1D solution and the 2D local zoom solution. The latters match perfectly with the "reference" solution.

These last two numerical tests show that in case of matching grids, a convergent coupling procedure based on the coupling source term $\Psi$ leads to a global solution as accurate as a full $2 \mathrm{D}$ solution.

Concerning the CPU times, let us remark that in the present simple configuration 
the faster approach is obviously to compute a full 2D solution (since the 1D part is negligible) and not the present superposition strategy. Roughly, the coupling algorithm which can require up to $p=4$ iterates (depending on the first guess) is more expansive (about $p$ times) than a full 2D simulation. In an operational context, difficulties and time costs are different. As mentionned in the introduction, the 1D model can be a complex network (with empirical laws calibrated by hand etc), its decomposition can be complex (1D data are not the same than $2 \mathrm{D}$ ones), the human time to decompose the $1 \mathrm{D}$ model can be important etc. Thus, in some operational contexts, the present superposition strategy has few advantages, including in a CPU time point of view.

\subsubsection{Subgrids case}

Now, the reference mesh is much finer, it has 10298 cells. The 2D zoom mesh is a restriction of this reference mesh. For the 1D model, we consider two coarser meshes: submesh either of a ratio $R_{\text {spac }}=\frac{\Delta x_{1 D}}{\Delta x_{2 D}}=2$ or of a ratio $R_{\text {spac }}=10$. Concerning time discretization, we keep the same time step for both models: $R_{t e m p}=\frac{\Delta t_{1 D}}{\Delta t_{2 D}}=1$.

Error due to the mismatching grids. As previously, as a first step, we proceed as follows:

- Initial conditions for both 1D and 2D models are extracted from the 2D "reference" solution,

- The boundary conditions of the 2D model is extracted from the 2D "reference" solution.

Therefore, we perform one iteration only of the Schwarz algorithm, the 2D zoom model reproduces the reference solution while the coupling source term in $1 \mathrm{D}$ equations include errors (with respect to the reference solution) due to the mismatching grids. In other words, errors are due to the integration of the source term over the 1D cells. Results obtained are presented in Fig. A.12.

Coupling with Schwarz algorithm. Starting from a "reasonable" initial condition, we deduce a first guess for the 2D incoming characteristics and we iterate the algorithm of Schwarz described in Fig. A.9. After convergence, the errors obtained are very similar than the latter, they are errors due to the grid mismatch.

Summary of the numerical results (Test 2). These numerical results show that after convergence, the coupling source term $\Psi$ defined in the present article leads to a global solution as accurate as a full $2 \mathrm{D}$ solution in case of matching grids, and leads to an accurate solution if grids are mismatching (for a sake of simplicity, only the subgrid has been implemented). This feature remains true with a more complex 
topography (see the toy test case presented in [12]).

\subsection{A practical point of view: superposition of a $2 D$ local zoom model vs full $2 D$}

In river hydraulics, the $1 \mathrm{D}$ areas (main channels of the river branches considered) can be complex networks. Furthermore, elaborating an operational 1D numerical model is a long and heavy task since data are not dense and precise (topography for example). Thus, in case of flooding, the present principle consisting to superpose locally a 2D model seems to be a good alternative to a re-definition of the whole "model" (that means to define a new full 2D model). In a discrete point of view, this principle of superposition becomes possible only if coupling term leading to an accurate and well-balanced global scheme can be defined.

In this last section, we illustrate both approaches: superposition of a $2 \mathrm{D}$ local zoom model vs full $2 \mathrm{D}$ with the following constraint: the $1 D$ main channel (mesh and topography) is given and cannot be changed. Then, we compare the following two computations:

- "Full 2D": we keep intact the 1D main channel (mesh and topography), and we extend the mesh inside the flood plain, see Fig. A.13 a). The 2D solver is applied all over the domain.

- "Superposition": Over the 1D model (mesh, topography, solver), we superpose the $2 \mathrm{D}$ model, using a finer mesh (those with $R_{\text {spac }}=10$ ) but with the constraint of meshing the lateral boundaries of the channel.

The results obtained with the full $2 \mathrm{D}$ solver are presented in Fig. A.13 b) and c) (legend "2D coarse-fine"). A comparison with Fig. A.12 shows that, as expected, we obtain a more precise solution using the principle of superposition. This result is obvious since in the superposition procedure, we have defined a $2 \mathrm{D}$ mesh which is finer next to the main channel $\left(R_{\text {spac }}=10\right)$ and similar next to the circle boundary. Nevertheless, this test case illustrates the following practical approach. If for any reason we must keep intact the $1 \mathrm{D}$ mesh, the present computations show that the superposition strategy leads to a more accurate solution compared to the full $2 \mathrm{D}$ solution based on the "existing" 1D mesh.

\section{Conclusion}

In this paper, we address the difficulty to couple and superpose numerically $1 \mathrm{D}$ and $2 \mathrm{D}$ shallow-water equations with non-flat topographies. We focus on the discrete definition of the coupling source term in the 1D equation, in order to obtain a wellbalanced and consistent approximation. To do so, we begin by writing a general form of the discrete the $1 \mathrm{D}$ and $2 \mathrm{D}$ problems by using a family of well-balanced 
finite volume solvers. We propose a discretization of the coupling source term that allows to use different finite volume schemes (explicit in time) for the $1 \mathrm{D}$ and the $2 \mathrm{D}$ problems. This allows, for example, to use a more accurate solver for 2D equations, and a less CPU time-consuming solver for the $1 \mathrm{D}$ equations, or the contrary. We prove that the proposed discretization is well-balanced independently of the choice of each solver. We present the problem for curvilinear 1D channel and mismatching grids. Our discretization technique introduces naturally an upwind definition of the tangential velocity at boundaries of the $1 \mathrm{D}$ channel. Thus, the global scheme remain stable whatever if lateral fluxes are locally incoming or outgoing. Numerical test cases show the efficiency and robustness of the discretization done, and show that the superposing approach is relevant. 


\section{A Derivation of the $2 \mathrm{D}$ source term in $1 \mathrm{D}$ equations}

We recall the derivation of the coupling source term in the $1 \mathrm{D}$ equations from the 3D Navier-Stokes equations. We refer to [12] for more details. We consider the incompressible Navier Stokes equations with no-slip boundary conditions at the bottom, and the kinematic equation at the free surface. We consider a 1D symmetric channel, centered in $x$-axis. By $b(x)$ we denote the channel width, $x \in[0, L]$. Lateral boundaries of the channel in the $(x, y)$-plan are defined by: $y=-b(x) / 2$ and $y=b(x) / 2$ (see Fig. A.2). We define the 1D wet cross section $S$ as follows: $S=\int_{b_{1}}^{b_{2}} \int_{z_{b}}^{z_{b}+h} d z d y$. For the sake of simplicity, we denote: $b_{1}(x)=-b(x) / 2$ and $b_{2}(x)=b(x) / 2$.

Mass conservation. Using standard notations, the mass conservation equation writes: $\frac{\partial u}{\partial x}+\frac{\partial v}{\partial y}+\frac{\partial w}{\partial z}=0$. By integration over $S$ we get:

$$
\underbrace{\int_{b_{1}}^{b_{2}} \int_{z_{b}}^{z_{b}+h} \frac{\partial u}{\partial x} d z d y}_{A}+\underbrace{\int_{b_{1}}^{b_{2}} \int_{z_{b}}^{z_{b}+h} \frac{\partial v}{\partial y} d z d y}_{B}+\underbrace{\int_{b_{1}}^{b_{2}} \int_{z_{b}}^{z_{b}+h} \frac{\partial w}{\partial z} d z d y}_{C}=0
$$

We apply the Leibnitz's integral rule and we use the no slip condition at bottom; this gives:

$$
\begin{aligned}
& A=\int_{b_{1}}^{b_{2}}\left(\frac{\partial}{\partial x} \int_{z_{b}}^{z_{b}+h} u d z-u_{S} \frac{\partial\left(z_{b}+h\right)}{\partial x}\right) d y \\
& =\frac{\partial}{\partial x} \int_{b_{1}}^{b_{2}} \int_{z_{b}}^{z_{b}+h} u d z d y-\left[\int_{z_{b}}^{z_{b}+h} u d z\right]_{y=b_{2}} \frac{\partial b_{2}}{\partial x}+\left[\int_{z_{b}}^{z_{b}+h} u d z\right]_{y=b_{1}} \frac{\partial b_{1}}{\partial x}-\underbrace{\int_{b_{1}}^{b_{2}} u_{S} \frac{\partial\left(z_{b}+h\right)}{\partial x} d y}_{C L 1} \\
& B=\int_{b_{1}}^{b_{2}}\left(\frac{\partial}{\partial y} \int_{z_{b}}^{z_{b}+h} v d z-v_{S} \frac{\partial\left(z_{b}+h\right)}{\partial y}\right) d y \\
& =\left[\int_{z_{b}}^{z_{b}+h} v d z\right]_{y=b_{2}}-\left[\int_{z_{b}}^{z_{b}+h} v d z\right]_{y=b_{1}}-\underbrace{\int_{b_{1}}^{b_{2}} v_{S} \frac{\partial\left(z_{b}+h\right)}{\partial y} d y}_{C L 2} \\
& C=\underbrace{\int_{b_{1}}^{b_{2}} w_{S} d y}_{C L 3}
\end{aligned}
$$

where $\left(u_{S}, v_{S}, w_{S}\right)^{T}$ denotes the surface velocity. We set: $Q=\int_{b_{1}}^{b_{2}} \int_{z_{b}}^{z_{b}+h} u d z d y$. Since the free surface boundary condition gives: $C L 1+C L 2+C L 3=\int_{b_{1}}^{b_{2}} \frac{\partial\left(z_{b}+h\right)}{\partial t} d y=$ 
$\partial_{t} S$, we obtain:

$$
(A .1) \Longleftrightarrow \frac{\partial S}{\partial t}+\frac{\partial Q}{\partial x}=K_{1}
$$

with:

$$
K_{1}=-\left(\left[\int_{z_{b}}^{z_{b}+h} v d z\right]_{b_{2}}-\left[\int_{z_{b}}^{z_{b}+h} u d z\right]_{b_{2}} \frac{\partial b_{2}}{\partial x}-\left[\int_{z_{b}}^{z_{b}+h} v d z\right]_{b_{1}}+\left[\int_{z_{b}}^{z_{b}+h} u d z\right]_{b_{1}} \frac{\partial b_{1}}{\partial x}\right)
$$

Without overflowing, these two terms vanish since $h=0$ at lateral boundaries (standard $1 \mathrm{D}$ shallow-water equations).

If $2 \mathrm{D}$ flow information is available at boundaries, $\left[\int_{z_{b}}^{z_{b}+h} u d z\right]_{b_{k}}$ and $\left[\int_{z_{b}}^{z_{b}+h} v d z\right]_{b_{k}}$, $k=1,2$, represent lineic discharges at boundaries in $x$-direction and $y$-direction respectively.

We denote by $\eta_{1}$ and $\eta_{2}$ the unit external normal vectors to the boundaries $y=$ $b_{1}(x)=-b(x) / 2$ and $y=b_{2}(x)=b(x) / 2$ respectively (see Fig. A.2), that is:

$$
\eta_{1}=\frac{1}{\delta_{b}}\left(\partial_{x} b_{1},-1\right)^{T} \quad \text { and } \quad \eta_{2}=\frac{1}{\delta_{b}}\left(-\partial_{x} b_{2}, 1\right)^{T}, \quad \text { with } \quad \delta_{b}=\sqrt{1+\left(\frac{\partial_{x} b}{2}\right)^{2}} .
$$

If the channel width variation is small then: $\delta_{b} \approx 1$. We set: $\delta_{b}=1$.

We set:

$$
q_{\eta_{i}}=\left[\int_{z_{b}}^{z_{b}+h} u d z\right]_{b_{i}}\left(\eta_{i}\right)_{1}+\left[\int_{z_{b}}^{z_{b}+h} v d z\right]_{b_{i}}\left(\eta_{i}\right)_{2}, \quad i=1,2 .
$$

Then we obtain:

$$
K_{1}=-\left(q_{\eta_{1}}+q_{\eta_{2}}\right)
$$

and the mass conservation equation is:

$$
\frac{\partial S}{\partial t}+\frac{\partial Q}{\partial x}=-\left(q_{\eta_{1}}+q_{\eta_{2}}\right)
$$

The values $q_{\eta_{i}}, i=1,2$, must be provided by a $2 \mathrm{D}$ model.

Momentum conservation. As previously, we integrate the 3D momentum equations over the 1D wet cross section $S$ and we use Leibniz integration rule. We consider the following hydrostatic pressure: $p=-\rho g\left(z-\left(H+\mathcal{Z}_{b}\right)\right)$ where $\left(H+\mathcal{Z}_{b}\right)$ is the mean transverse water elevation $\left(\mathcal{Z}_{b}\right.$ is the mean transverse topography i.e. it is the 
so-called uni-dimensional topography). Then, we obtain:

$$
\frac{\partial Q}{\partial t}+\frac{\partial}{\partial x}\left(\beta \frac{Q^{2}}{S}\right)+g S \frac{\partial\left(H+\mathcal{Z}_{b}\right)}{\partial x}=K_{2}
$$

where $\beta$ is a so-called Boussinesq coefficient, $\beta=\frac{S}{Q^{2}} \int_{S} u^{2} d S$, and:

$$
K_{2}=-\left[\int_{z_{b}}^{z_{b}+h} u v d z\right]_{b_{1}}^{b_{2}}-\left[\int_{z_{b}}^{z_{b}+h} u^{2} d z\right]_{b_{1}} \frac{\partial b_{1}}{\partial x}+\left[\int_{z_{b}}^{z_{b}+h} u^{2} d z\right]_{b_{2}} \frac{\partial b_{2}}{\partial x}
$$

Again, without overflowing (standard 1D St-Venant equations), this term vanishes since $h=0$ at lateral boundaries.

Let us notice that we neglected the friction term derived from the viscous term in the 3D Navier-Stokes equations.

If $(u, v)$ do not depend on $z$ on boundaries $b_{1}$ and $b_{2}$, then the source term $K_{2}$ writes:

$$
K_{2}=-\left(\left.u\right|_{b_{1}} q_{\eta_{1}}+\left.u\right|_{b_{2}} q_{\eta_{2}}\right)
$$

If $u$ is constant over the cross section then $\beta=1$. We assume that $\beta=1$.

Finally, we obtain the following shallow momentum equation with source term:

$$
\frac{\partial Q}{\partial t}+\frac{\partial}{\partial x}\left(\frac{Q^{2}}{S}\right)+g S \frac{\partial\left(H+\mathcal{Z}_{b}\right)}{\partial x}=-\left(\left.u\right|_{b_{1}} q_{\eta_{1}}+\left.u\right|_{b_{2}} q_{\eta_{2}}\right)
$$

Rectangular cross-section case. For the sake of simplicity, we consider rectangular cross sections in the main channel, then: $S=b H$, where $b$ is the channel width. If we define the pressure term, see e.g. [9]:

$$
P=g S \frac{H}{2}=g \frac{S^{2}}{2 b}
$$

then we have: $\frac{\partial P}{\partial x}=g\left(\frac{\partial b}{\partial x} \frac{H^{2}}{2}+S \frac{\partial H}{\partial x}\right)$.

Finally the equations (A.3) and (A.5) can be written as an hyperbolic system with source terms as follows:

$$
\left\{\begin{array}{l}
\frac{\partial S}{\partial t}+\frac{\partial Q}{\partial x}=-\left(q_{\eta_{1}}+q_{\eta_{2}}\right) \\
\frac{\partial Q}{\partial t}+\frac{\partial}{\partial x}\left(\frac{Q^{2}}{S}+P\right)-g \frac{\partial b}{\partial x} \frac{H^{2}}{2}+g S \frac{\partial \mathcal{Z}_{b}}{\partial x}=-\left(\left.u\right|_{b_{1}} q_{\eta_{1}}+\left.u\right|_{b_{2}} q_{\eta_{2}}\right)
\end{array}\right.
$$




\section{Acknowledgements}

The authors would like to thank particularly a reviewer for his constructive comments. This work has been partially supported by the Spanish Government Research projects MTM2006-01275, MTM2009-07719 and P06-RNM-01594.

\section{References}

[1] E. Blayo, L. Debreu. Revisiting open boundary conditions from the point of view of characteristic variables. Ocean Modelling, vol 9, pp 231-252 (2005).

[2] M.J. Castro, T. Chacón, E.D. Fernández-Nieto, C. Parés On well-balanced finite volume methods for non-conservative non-homogeneous systems. SIAM J. Scientific Comp., V. 29, n. 3, 1093-1126 (2007).

[3] M.J. Castro, A.M. Ferreiro, J.A. García, J.M. González, J. Macías, C. Parés, M.E. Vázquez, On the numerical treatment of wet/dry fronts in shallow flows: application to one-layer and two-layer systems. Match Comp. Model. 42 (3-4): 419-439 (2005).

[4] M.J. Castro, T. Chacón Rebollo,E.D. Fernández-Nieto, J.M. González-Vida, C. Parés, Well-Balanced Finite Volume schemes for 2D non-homogeneous hyperbolic systems. Application to the Dam-break of Aznalcóllar. Comput. Methods Appl. Mech. Engrg. 197: 3932-3950 (2008).

[5] T. Chacón Rebollo, A. Domínguez, E.D. Fernández-Nieto A family of stable numerical solvers for the shallow water equations with source terms Comput. Methods Appl. Mech. Engrg. 192, 203-225 (2003).

[6] J.A. Cunge, F.M.J. Holly, A. Verwey. Practical aspects of computational river hydraulics. Vol 3 of Monographs and surveys in water resources engineering, Pitman, London (1980).

[7] E.D. Fernández-Nieto, D. Bresch, J. Monnier A consistent intermediate wave speed for a well-balanced HLLC solver C. R. Acad. Sci. Paris, Ser. I 346, pp 795-800 (2008).

[8] I. Y. Gejadze, J. Monnier. On a 2D zoom for $1 D$ shallow-water model: coupling and data assimilation. Comp. Meth. Appl. Mech. Eng. Vol. 196, issues 45-48, pp 4628-4643 (2007).

[9] N. Goutal, F. Maurel. A finite volume solver for $1 D$ shallow-water equations applied to an actual river. Int. J. Numer. Meth. Fluids, vol 38, pp 1-19 (2002).

[10] M. Honnorat, J. Marin, J. Monnier. DassFlow : Data Assimilation for River Flows. http://www-gmm.insa-toulouse.fr/ monnier/DassFlow

[11] M. Honnorat, J. Marin, J. Monnier, X. Lai. Dassflow v1.0: a variational data assimilation software for $2 D$ river flows. INRIA Research Report number 6150 (2007). 
[12] J. Marin, J. Monnier. Superposition of local zoom model and simultaneous calibration for 1D-2D shallow-water flows. Math. Comput. Simul. 80, 547-560 (2009).

[13] E. Miglio, S. Perotto, F. Saleri. Model coupling techniques for free-surface flow problems: Part I. Nonlinear Analysis, vol 63, pp 1885-1896 (2005).

[14] H. Roux. Estimation de paramtres en hydraulique fluviale, partir de donnes caractristiques de limagerie arienne. PhD Thesis, INP-Toulouse, 2004.

[15] G. Steinebach, S. Rademacher, P. Rentrop, M. Schulz. Mechanisms of coupling in river flow simulation systems. J. Comput. Appl. Math., vol 168, pp 459-470 (2004).

[16] E F. Toro. Shock-Capturing Methods for Free-Surface Shallow Flows. Wiley and Sons, Ltd (2001). 


\section{List of Figures}

A.1 Modeling outline: a global 1D model with superposed local 2D models.

A.2 Up: definition of the $1 \mathrm{D}$ channel in the $2 \mathrm{D}$ domain. Down: $1 \mathrm{D}$ cross section with overflowing

A.3 1D model. a) Wet cross section ; b) Top view of one 1D cell in the main channel

A.4 2D cell with definition of $q_{\eta_{i}}$ and $u_{t_{i}}$

A.5 Notation, finite control volumes

A.6 Simplest case and matching grids.

A.7 General domain with matching grids.

A.8 Information transfer. 1D main channel $\Omega_{1}, 2 \mathrm{D}$ flood plain $\Omega_{2}$, overflowing boundaries $\Gamma_{1}, \Gamma_{2}$

A.9 Coupling algorithm based on a Schwarz method.

A.10 Test case: a) Bathymetry and mesh of 2274 cells, b) "Reference" steady state solution (velocity field).

A.11 Matching grids case. Comparison of velocity values $(u)$ in the 1D main channel (common area) and after Schwarz algorithm convergence. a) 2D reference solution and values computed by the 1D model and by the local 2D zoom model; b) Differences in percent.

A.12 Subgrids case. Comparison of velocity values $(u)$ in the $1 \mathrm{D}$ main channel (common area) and after Schwarz algorithm convergence. a) $R_{\text {spac }}=2.2 \mathrm{D}$ reference solution and values computed by the $1 \mathrm{D}$ model and by the local 2D zoom model; b) Differences in percent. c) $R_{\text {spac }}=10.2 \mathrm{D}$ reference solution and values computed by the $1 \mathrm{D}$ model and by the local 2D zoom model; d) Differences in percent.

A.13 Superposition vs full 2D. a) The full 2D mesh is defined from the 1D mesh in the channel $(R=1)$. b) Velocity $u$ in the main channel: "full 2D" solution $(R=1$, legend "2D coarse-fine") and the $2 \mathrm{D}$ coupled solution with $R_{\text {spac }}=10$ (legend "reference"). c) Differences in percent. 


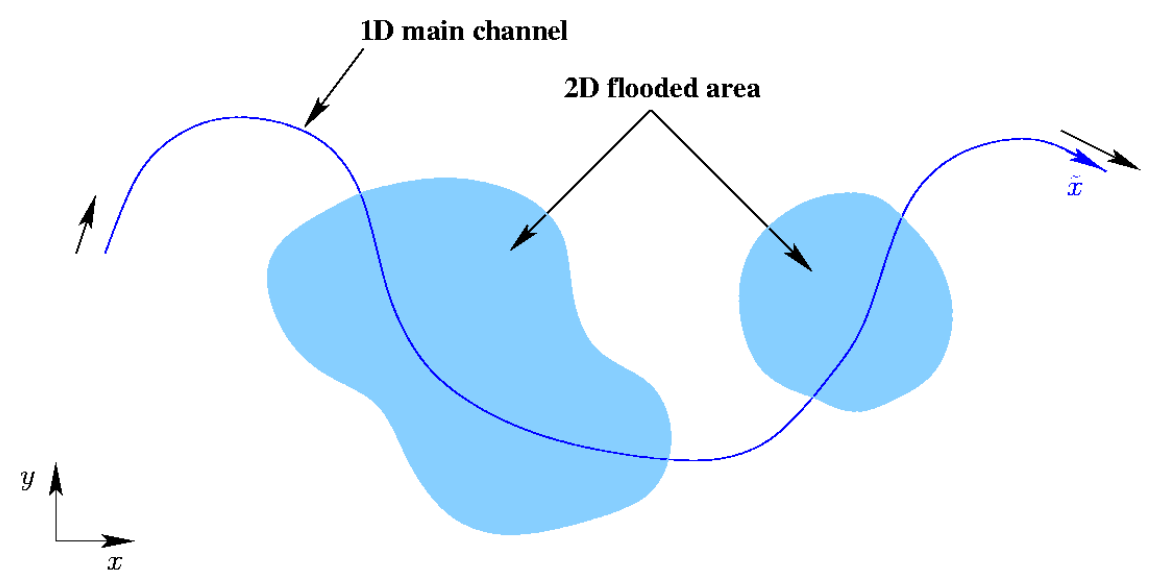

Fig. A.1. Modeling outline: a global 1D model with superposed local 2D models.

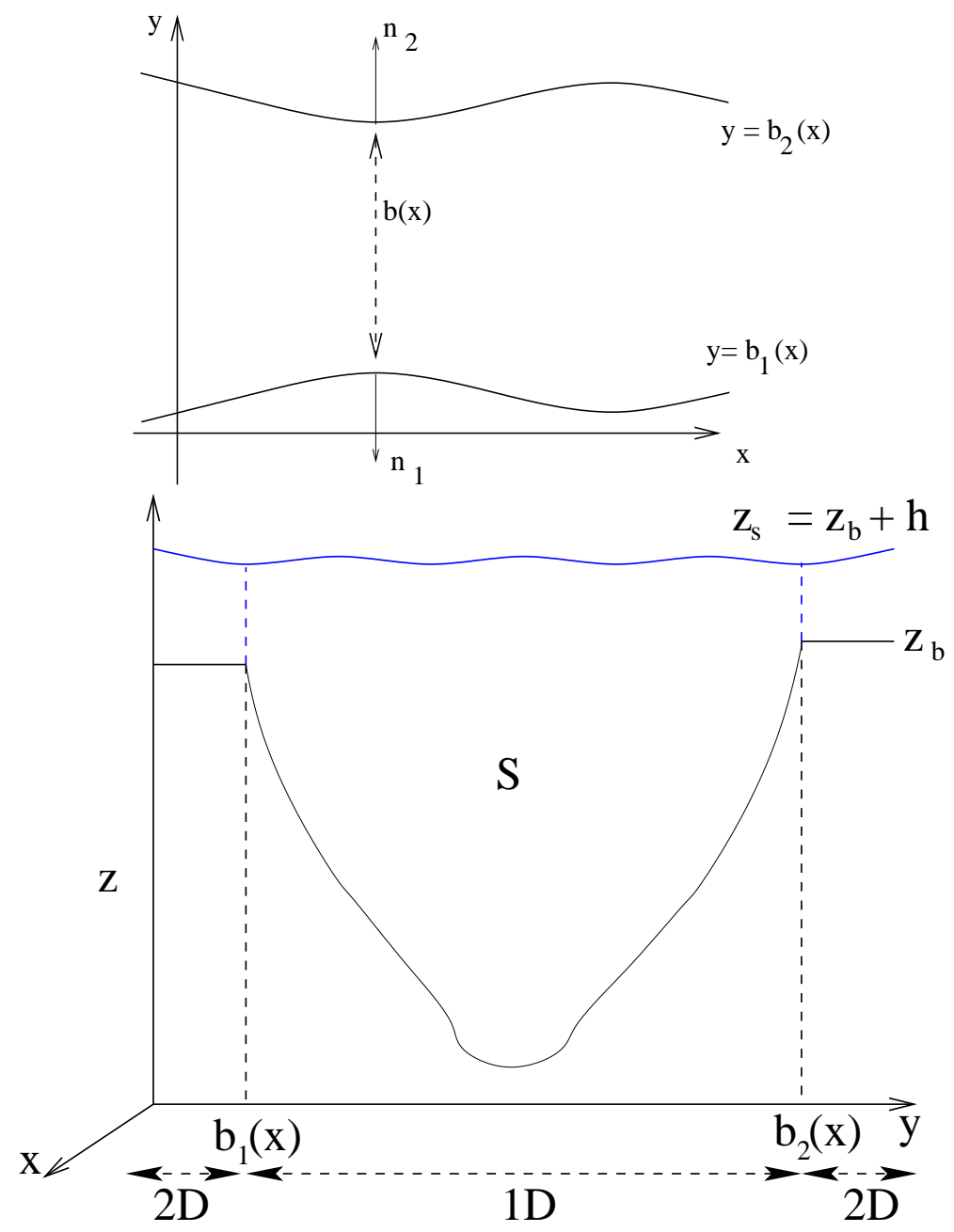

Fig. A.2. Up: definition of the 1D channel in the 2D domain. Down: 1D cross section with overflowing 

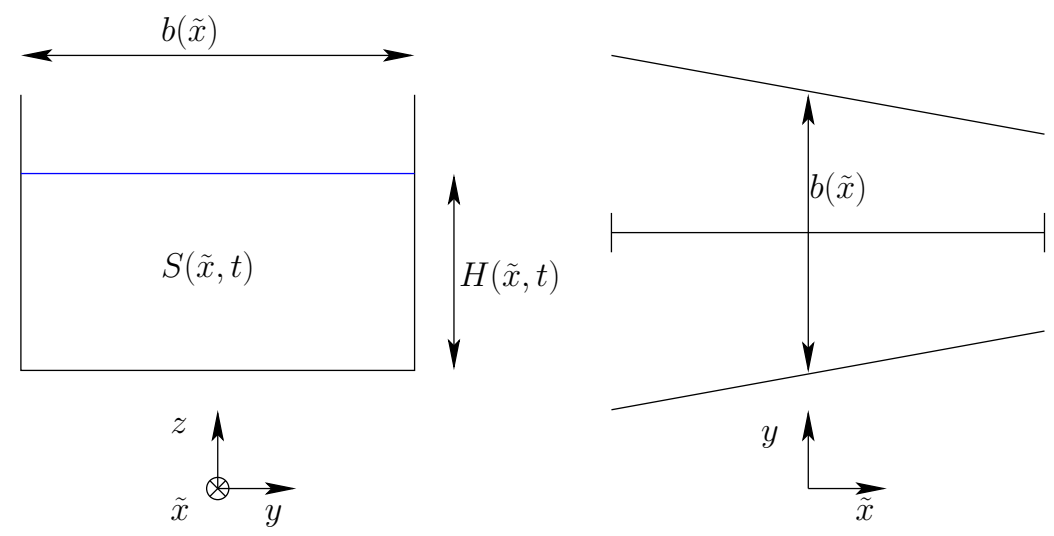

Fig. A.3. 1D model. a) Wet cross section ; b) Top view of one 1D cell in the main channel

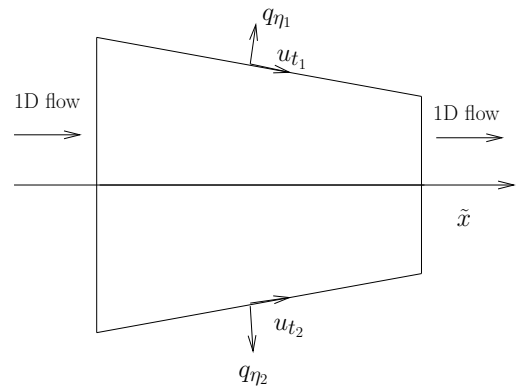

Fig. A.4. 2D cell with definition of $q_{\eta_{i}}$ and $u_{t_{i}}$

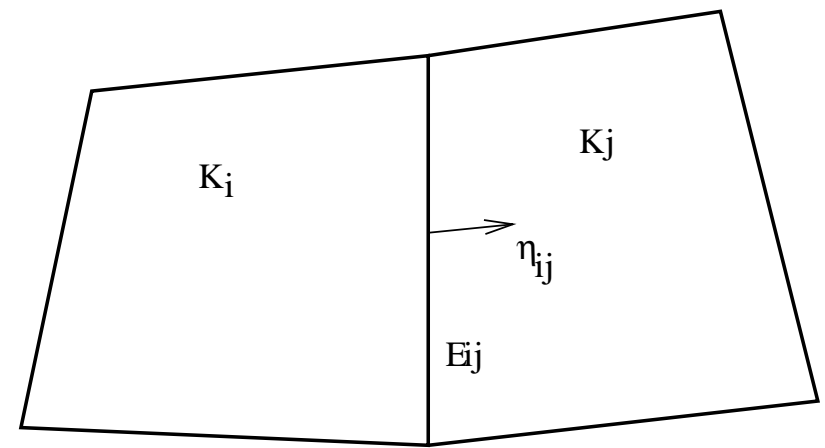

Fig. A.5. Notation, finite control volumes 


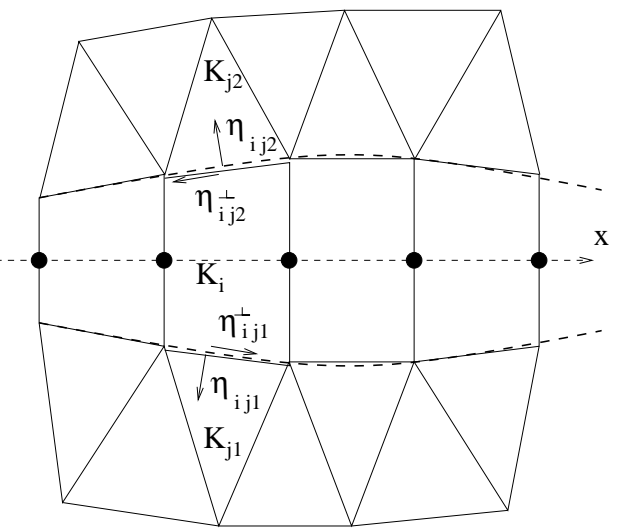

Fig. A.6. Simplest case and matching grids.

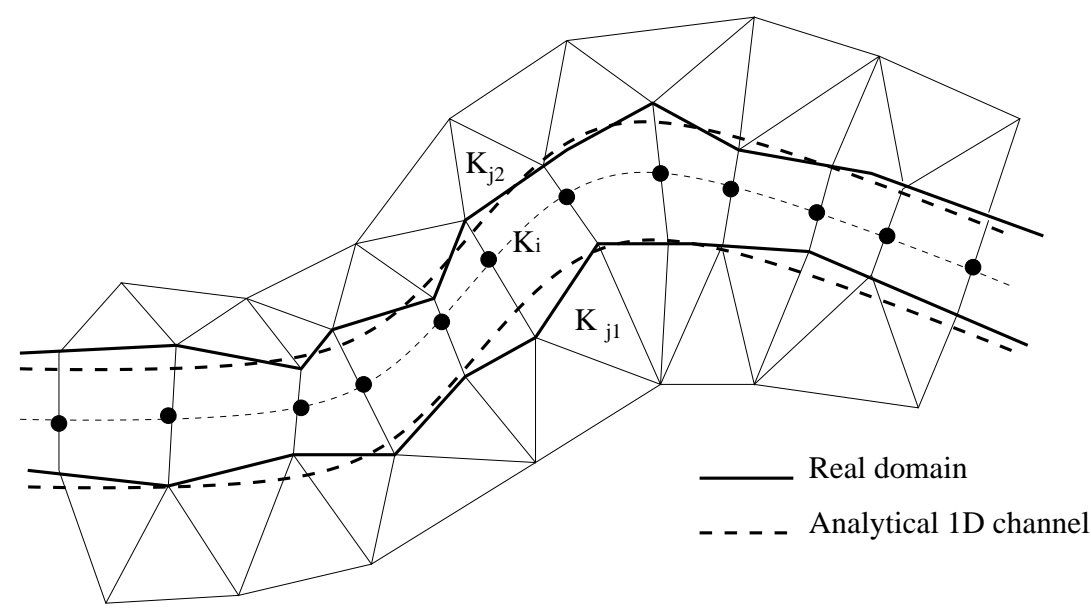

Fig. A.7. General domain with matching grids.

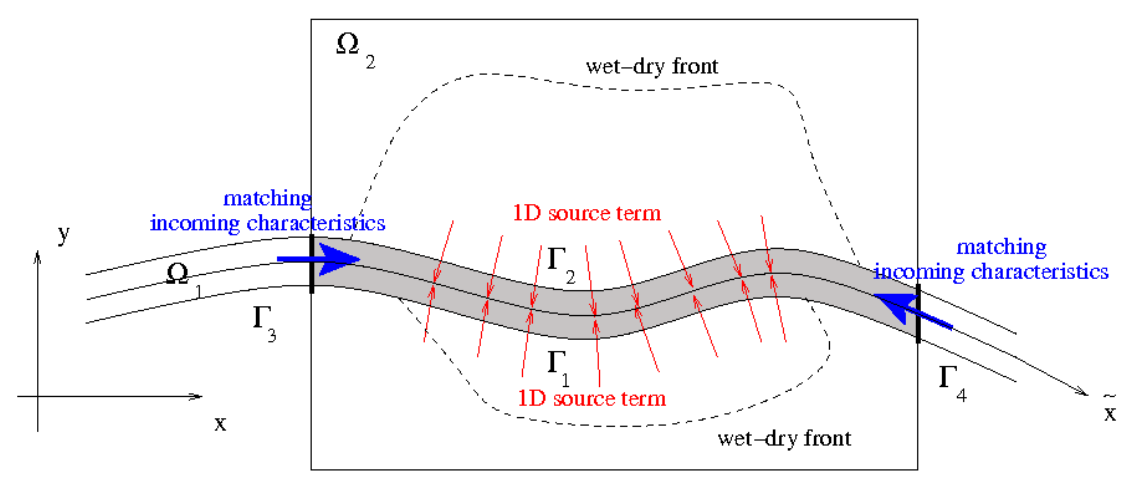

Fig. A.8. Information transfer. 1D main channel $\Omega_{1}, 2 \mathrm{D}$ flood plain $\Omega_{2}$, overflowing boundaries $\Gamma_{1}, \Gamma_{2}$ 


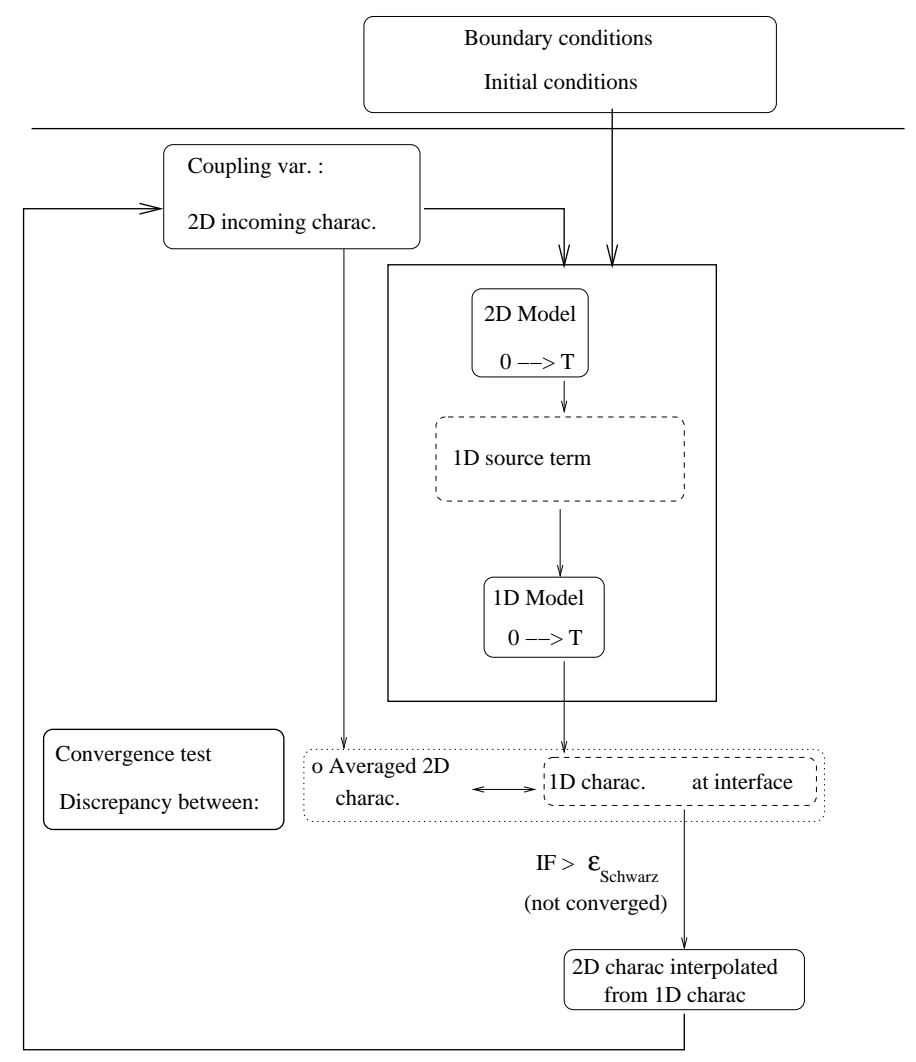

Fig. A.9. Coupling algorithm based on a Schwarz method.

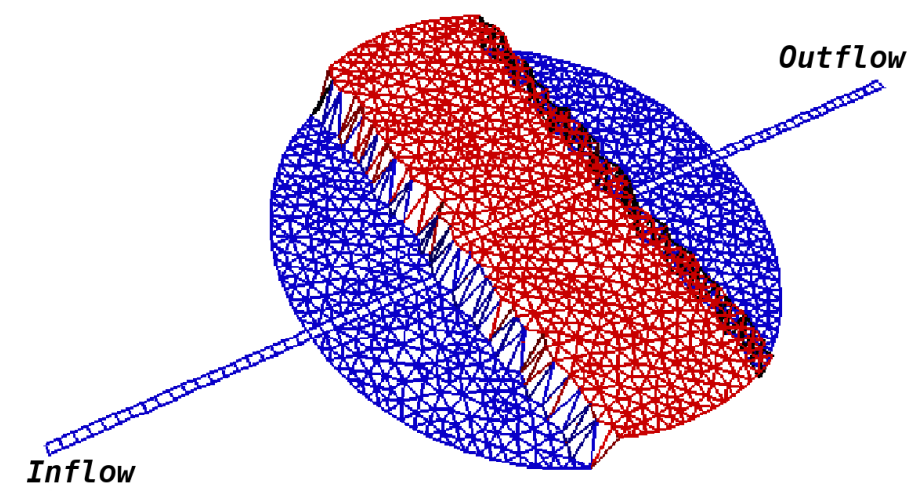

a)

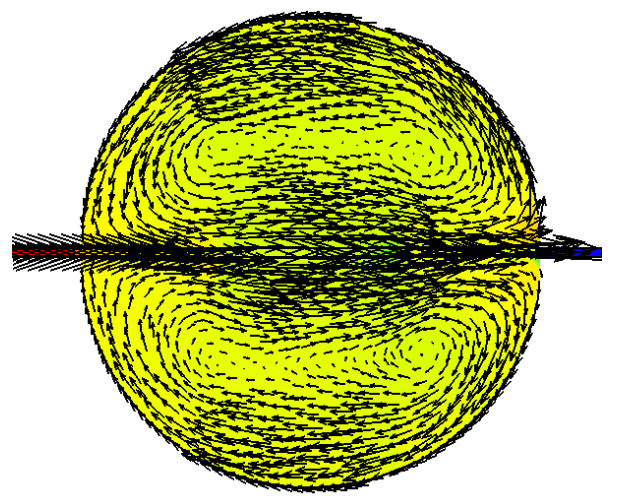

b)

Fig. A.10. Test case: a) Bathymetry and mesh of 2274 cells, b) "Reference" steady state solution (velocity field). 


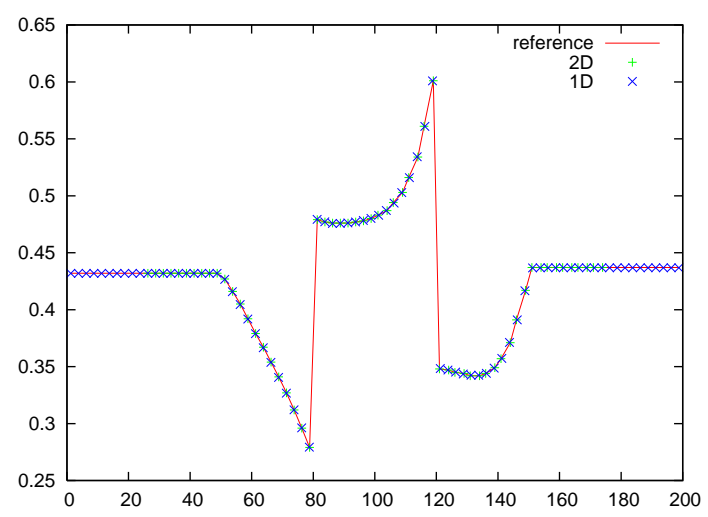

a)

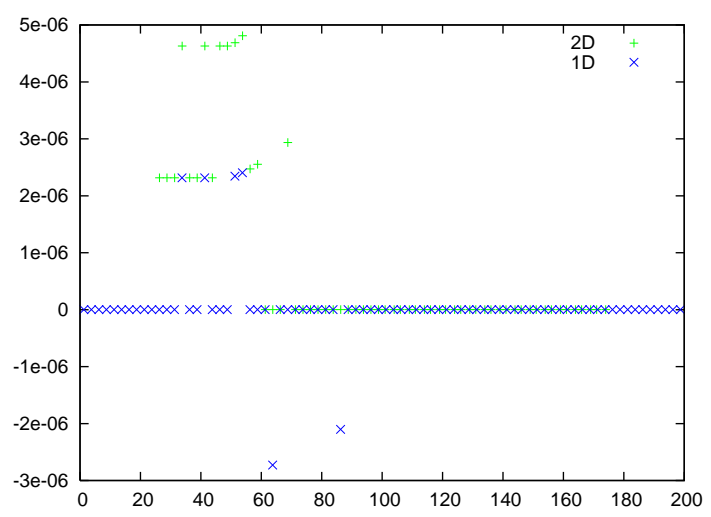

b)

Fig. A.11. Matching grids case. Comparison of velocity values $(u)$ in the $1 \mathrm{D}$ main channel (common area) and after Schwarz algorithm convergence. a) 2D reference solution and values computed by the $1 \mathrm{D}$ model and by the local $2 \mathrm{D}$ zoom model; b) Differences in percent. 


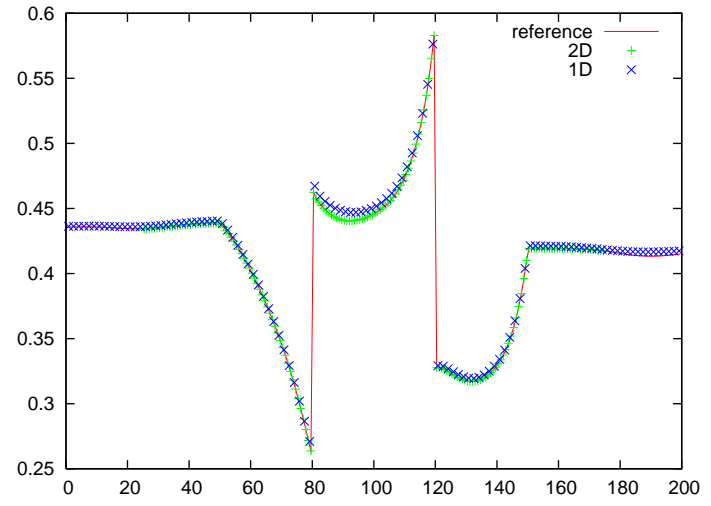

a)

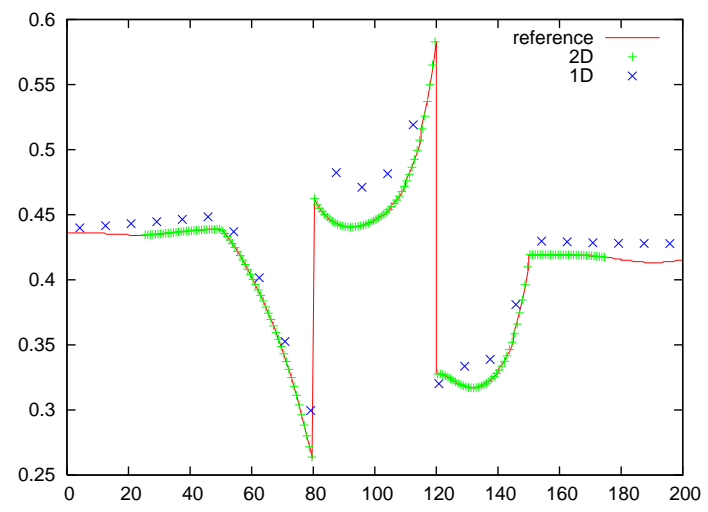

c)

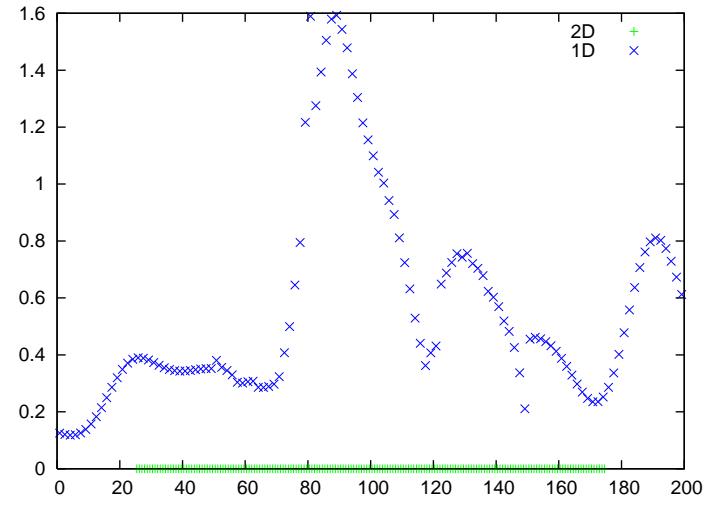

b)

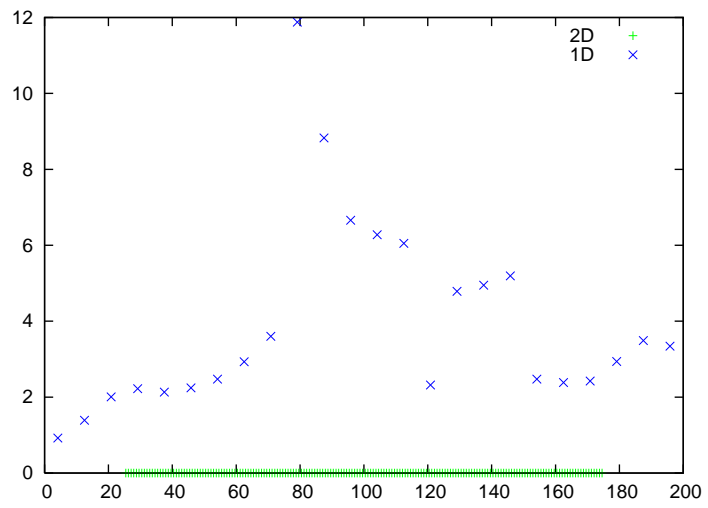

d)

Fig. A.12. Subgrids case. Comparison of velocity values $(u)$ in the 1D main channel (common area) and after Schwarz algorithm convergence. a) $R_{\text {spac }}=2$. $2 \mathrm{D}$ reference solution and values computed by the 1D model and by the local 2D zoom model; b) Differences in percent. c) $R_{\text {spac }}=10.2 \mathrm{D}$ reference solution and values computed by the $1 \mathrm{D}$ model and by the local 2D zoom model; d) Differences in percent. 


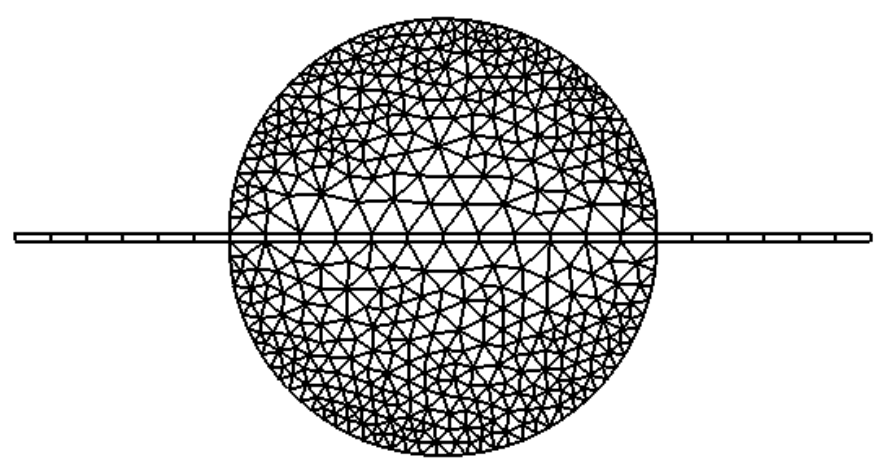

a)

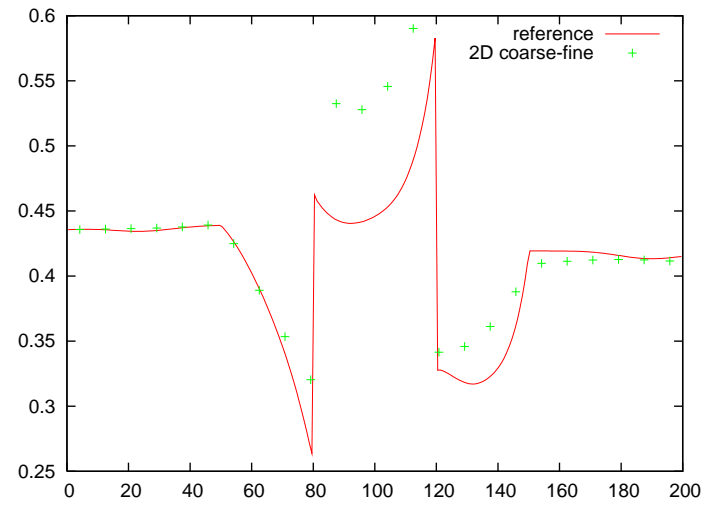

b)

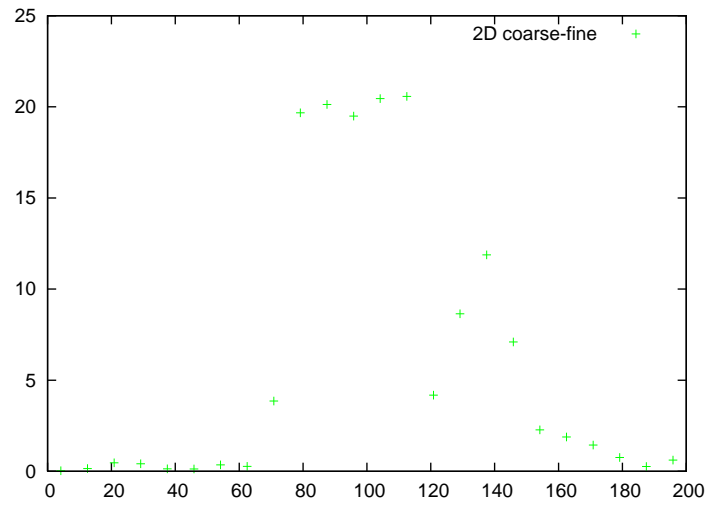

c)

Fig. A.13. Superposition vs full 2D. a) The full $2 \mathrm{D}$ mesh is defined from the $1 \mathrm{D}$ mesh in the channel $(R=1)$. b) Velocity $u$ in the main channel: "full $2 \mathrm{D}$ " solution $(R=1$, legend "2D coarse-fine") and the $2 \mathrm{D}$ coupled solution with $R_{\text {spac }}=10$ (legend "reference"). c) Differences in percent. 